\title{
Review Article \\ Protocol Variations and Six-Minute Walk Test Performance in Stroke Survivors: A Systematic Review with Meta-Analysis
}

\author{
A. Dunn, ${ }^{1,2}$ D. L. Marsden, ${ }^{1,2,3}$ E. Nugent, ${ }^{1}$ P. Van Vliet, ${ }^{1,2}$ N. J. Spratt, ${ }^{1,2,3}$ \\ J. Attia, ${ }^{1,2}$ and R. Callister ${ }^{1,2}$ \\ ${ }^{1}$ University of Newcastle, Callaghan, NSW 2308, Australia \\ ${ }^{2}$ Hunter Medical Research Institute, New Lambton Heights, NSW 2305, Australia \\ ${ }^{3}$ Hunter New England Local Health District, New Lambton Heights, NSW 2305, Australia
}

Correspondence should be addressed to A. Dunn; ashlee.dunn@uon.edu.au

Received 30 September 2014; Revised 18 December 2014; Accepted 18 December 2014

Academic Editor: Graeme Hankey

Copyright (C) 2015 A. Dunn et al. This is an open access article distributed under the Creative Commons Attribution License, which permits unrestricted use, distribution, and reproduction in any medium, provided the original work is properly cited.

\begin{abstract}
Objective. To investigate the use of the six-minute walk test (6MWT) for stroke survivors, including adherence to 6MWT protocol guidelines and distances achieved. Methods. A systematic search was conducted from inception to March 2014. Included studies reported a baseline (intervention studies) or first instance (observational studies) measure for the 6MWT performed by stroke survivors regardless of time after stroke. Results. Of 127 studies (participants $n=6,012$ ) that met the inclusion criteria, 64 were also suitable for meta-analysis. Only 25 studies made reference to the American Thoracic Society (ATS) standards for the 6MWT, and 28 reported using the protocol standard $30 \mathrm{~m}$ walkway. Thirty-nine studies modified the protocol walkway, while 60 studies did not specify the walkway used. On average, stroke survivors walked $284 \pm 107 \mathrm{~m}$ during the 6MWT, which is substantially less than healthy age-matched individuals. The meta-analysis identified that changes to the ATS protocol walkway are associated with reductions in walking distances achieved. Conclusion. The 6MWT is now widely used in stroke studies. The distances achieved by stroke patients indicate substantially compromised walking ability. Variations to the standard $30 \mathrm{~m}$ walkway for the $6 \mathrm{MWT}$ are common and caution should be used when comparing the values achieved from studies using different walkway lengths.
\end{abstract}

\section{Introduction}

Compromised walking ability is a functional limitation significantly associated with poorer community integration following stroke [1] and improving walking capacity and endurance is often a key goal of stroke rehabilitation [2-5]. Functional walking tests, such as the 6MWT, were originally developed in the 1960s and used to assess people with cardiovascular and respiratory disease [6, 7]. More recently, the $6 \mathrm{MWT}$ has been used to characterise and monitor changes in walking capacity following stroke. The test is commonly used as a measure of walking endurance and is a significant predictor of community ambulation and integration in stroke survivors [8].

In 2002, the American Thoracic Society (ATS) published guidelines for the 6MWT [9] with the objective of standardising the protocol to encourage further application of the $6 \mathrm{MWT}$ and allow direct comparisons among different studies and populations. The ATS guidelines include test indications and contraindications, safety measures, and a step-by-step protocol and provide assistance with clinical interpretation. Key components of the protocol include the test location, walkway length, measurements, and instructions. According to the ATS protocol, the test should be performed on a flat, enclosed (indoor) walkway $30 \mathrm{~m}$ in length. This protocol requires $180^{\circ}$ turns at either end of the walkway and additional space for turning is required. The guidelines advise that shorter walkway lengths require more directional changes and can reduce the distances achieved [9]. It is likely that the influence of directional changes may be amplified in the stroke population, who characteristically have impaired balance, asymmetrical gait patterns, and altered responses for turn preparation $[8,10,11]$. Conversely, reducing the number of directional changes may increase the distance achieved [12]. 
The aims of this systematic review were to synthesise the current literature that used the 6MWT and to investigate (1) the extent of its use in stroke survivors; (2) the characteristics of the stroke survivor populations studied; (3) the adherence to the ATS standard protocol; (4) the distances achieved; and (5) the influences of protocol modification and factors such as age, gender, disability, and time after stroke on distances achieved.

\section{Methods}

The conduct and reporting of this review was guided by the Preferred Reporting Items for Systematic Reviews and MetaAnalyses (PRISMA) statement and the Meta-Analysis of Observational Studies in Epidemiology (MOOSE) statement [13].

2.1. Search Strategy and Selection Criteria. A systematic computer-based search was undertaken of the databases MEDLINE, CINAHL, EMBASE, PsycINFO, AMED, SPORTDiscus, and COCHRANE from inception to 24th of March, 2014. The search strategy used for MEDLINE is outlined in Table 1 and was adapted to suit each database as required. Studies were deemed eligible if they were published in English, in peer-reviewed journals, and included the distance walked during the $6 \mathrm{MWT}$ by stroke survivors during the baseline (intervention studies) or first instance (observational studies). The World Health Organisation [14] defines stroke as "a focal (or at times global) neurological impairment of sudden onset, and lasting more than 24 hours (or leading to death), and of presumed vascular origin" and must have been diagnosed clinically. Both ischemic and haemorrhagic stroke were included at any time after stroke. Studies reporting data from mixed neurological groups that included people after stroke were excluded. Theses and articles published in abstract form only, including conference proceedings, were also excluded.

2.2. Selection of Studies. The author A. Dunn identified and obtained abstracts from the relevant studies based on title and classified each study as being a possible inclusion or definite exclusion according to the first inclusion criterion the study failed to meet. Studies were excluded if they were a subset from a larger study using the same participants, if the distance achieved on the 6MWT was not presented as text but rather a graph or chart, or if the $6 \mathrm{MWT}$ distance was not reported. Full-text versions of all possible inclusion studies were then retrieved and reviewed by the author A. Dunn who classified them as "include," "exclude," or "unsure." This process was then independently conducted by the second reviewer E. Nugent. For instances where there was uncertainty or disagreement between authors, a consensus decision was reached through discussion and the involvement of a third reviewer, R. Callister, if necessary.

2.3. Data Extraction and Synthesis. Author A. Dunn then extracted the following variables from included studies:
TABLE 1: Search strategy used for MEDLINE.

\begin{tabular}{|c|c|}
\hline 1 & $\begin{array}{l}\text { Cerebrovascular Disorders.mp. or exp Cerebrovascular } \\
\text { Disorders }\end{array}$ \\
\hline 2 & Stroke.mp. or exp Stroke/ \\
\hline 3 & (cerebral or cerebellar or brainstem or vertebrobasilar).mp. \\
\hline 4 & (infarct ${ }^{*}$ or ischemia or thrombo* or embol $\left.{ }^{*}\right) \cdot m p$. \\
\hline 5 & 3 and 4 \\
\hline 6 & (cerebral or brain or subarachnoid or intracerebral).mp. \\
\hline 7 & $\begin{array}{l}\text { (haemorrhage or haematoma or bleed }{ }^{*} \text { or haemorrhage or } \\
\text { hematoma).mp. }\end{array}$ \\
\hline 8 & 6 and 7 \\
\hline 9 & 1 or 2 or 5 or 8 \\
\hline 10 & $6 \mathrm{MWT}$ \\
\hline 11 & Six minute walk* \\
\hline 12 & 6 minute walk* \\
\hline 13 & 10 or 11 or 12 \\
\hline 14 & 9 and 13 \\
\hline
\end{tabular}

(i) study characteristics: year published, participant numbers, and gender ratio;

(ii) participant characteristics: age, gender, time since stroke, and disability score;

(iii) 6MWT: distance achieved, protocol used, assistance provided, assistive devices used, and instructions given.

2.4. Quantitative Analysis of Adherence to ATS Protocol Guidelines. To date, there is no standardised approach to assessing the quality of reporting of observational studies such as adherence to protocol guidelines. As this is a systematic review looking only at baseline values rather than interventions, assessment of conventional methodological study quality is not applicable. Therefore, for the purpose of this review, a unique two-point scale was designed. Points were awarded as follows: one point for describing the protocol used and one point for referring to the ATS standards for the $6 \mathrm{MWT}$. Therefore, a study could score zero, one, or two points unless it was published prior to the ATS standards $(n=3)$ in which case it could only achieve a score of zero or one.

2.5. Meta-Analysis. A meta-analysis was conducted to examine the influence of the 6MWT protocol variations, as well as age (continuous, years), gender (proportion of male participants in the study), physical disability score (converted to a continuous $z$-score), and time since stroke (continuous, months), on the distance walked. Studies were excluded from the meta-analysis if they did not perform the 6MWT indoors, on a flat walkway, with usual walking device, or if the walkway length was not described. Included studies were pooled into three groups based on the walkway length used: $<30 \mathrm{~m},=30 \mathrm{~m}$, and $>30 \mathrm{~m}$ shuttles. Studies where the test was performed on an oval or rectangular track were pooled together to create a fourth "continuous walkway" group. The ATS standards state $30 \mathrm{~m}$ or $100 \mathrm{ft}$ walkway, which converts 


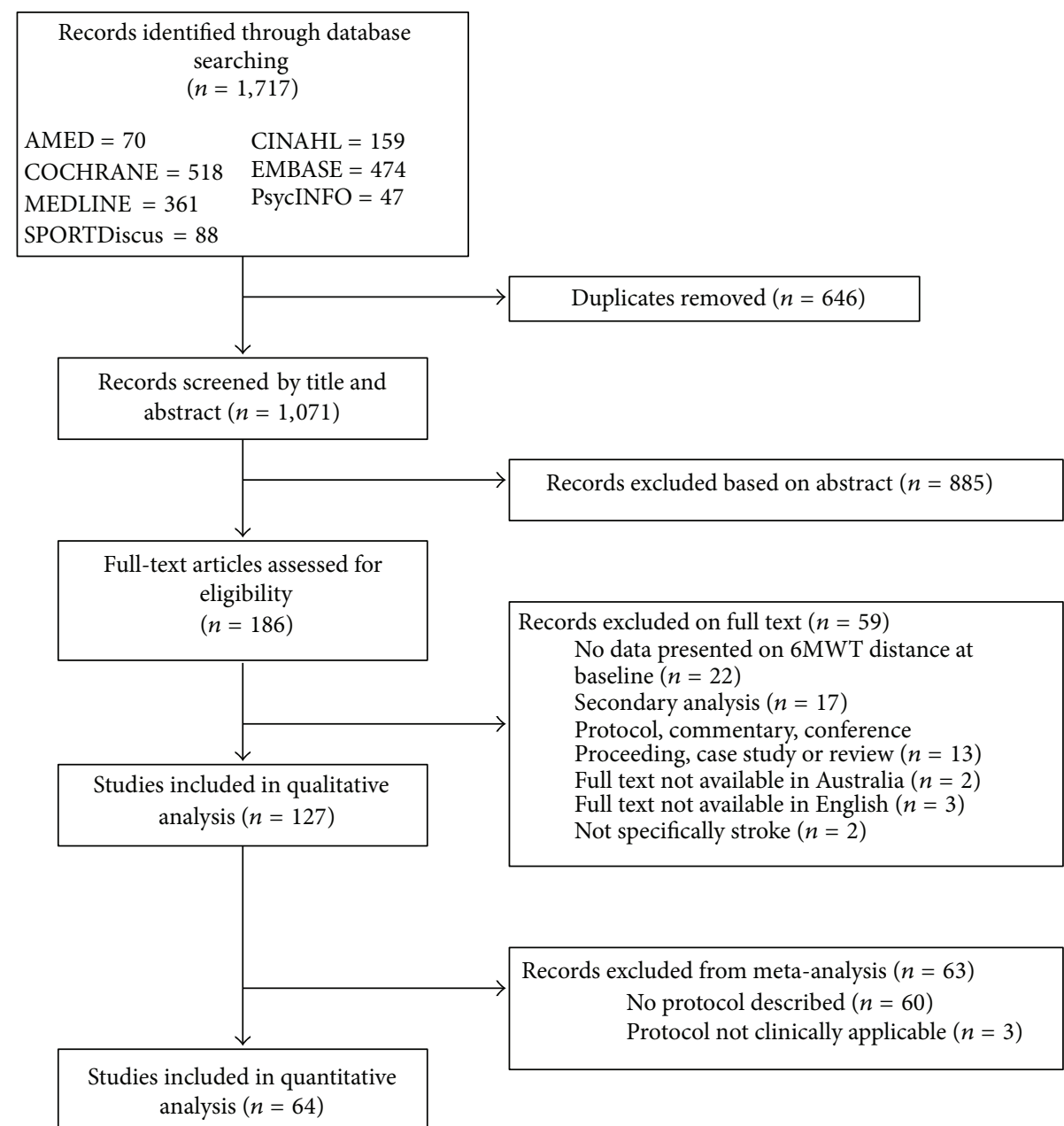

FIGURE 1: PRISMA flow diagram.

to $30.5 \mathrm{~m}$. Any studies using a $30.5 \mathrm{~m}$ walkway were therefore included in the $=30 \mathrm{~m}$ group. In studies that reported medians (IQRs), the medians were used as means and IQRs were converted to SDs by dividing the reported IQRs by 1.35; these approaches assume symmetrical distributions. A randomeffects meta-regression was conducted where the square of each study's standard error was used as fixed values of the sampling variance. Statistical significance was accepted at the level of $\alpha \leq 0.05$. A second meta-regression was also conducted to examine the effects of age, gender, disability, and time since stroke in only those studies that used the $30 \mathrm{~m}$ walkway protocol.

\section{Results}

The search across seven databases yielded 1,717 citations from which 127 articles were identified for inclusion in the review. Figure 1 details the flow of studies and reasons for exclusion throughout the selection process. Table 2 summarises the study characteristics, participant characteristics, and 6MWT results. Of the included studies the first paper to use the $6 \mathrm{MWT}$ in stroke survivors was reported in 1998 [39]. The use of the test has since increased rapidly, with 11 papers published from 2000 to 2004,48 papers published from 2005 to 2009, and 67 papers published from 2010 onward. Most (98\%) papers were published since the publication of the ATS guidelines in 2002 .

3.1. Participant Characteristics. The 127 studies reported on a collective sample size of 6,012 participants, including 3,654 males (61\%), 2,188 females (36\%), and 170 (3\%) not specified. The participant eligibility criteria reported in studies included the following: participants greater than 6 months after stroke ( $n=40$ studies), no significant cognitive or communicative issues ( $n=85$ studies), mild or no cardiovascular or pulmonary problems ( $n=66$ studies), no orthopaedic or musculoskeletal problems or pain ( $n=57$ studies), no other neurological conditions ( $n=42$ studies), and participants able to meet a specified minimal or maximal walking speed either overground or on a treadmill ( $n=17$ studies). Only 77 studies reported being ambulant with or without assistance as eligibility criteria; however a further nine studies reported a minimal walking speed and four studies report ability to 


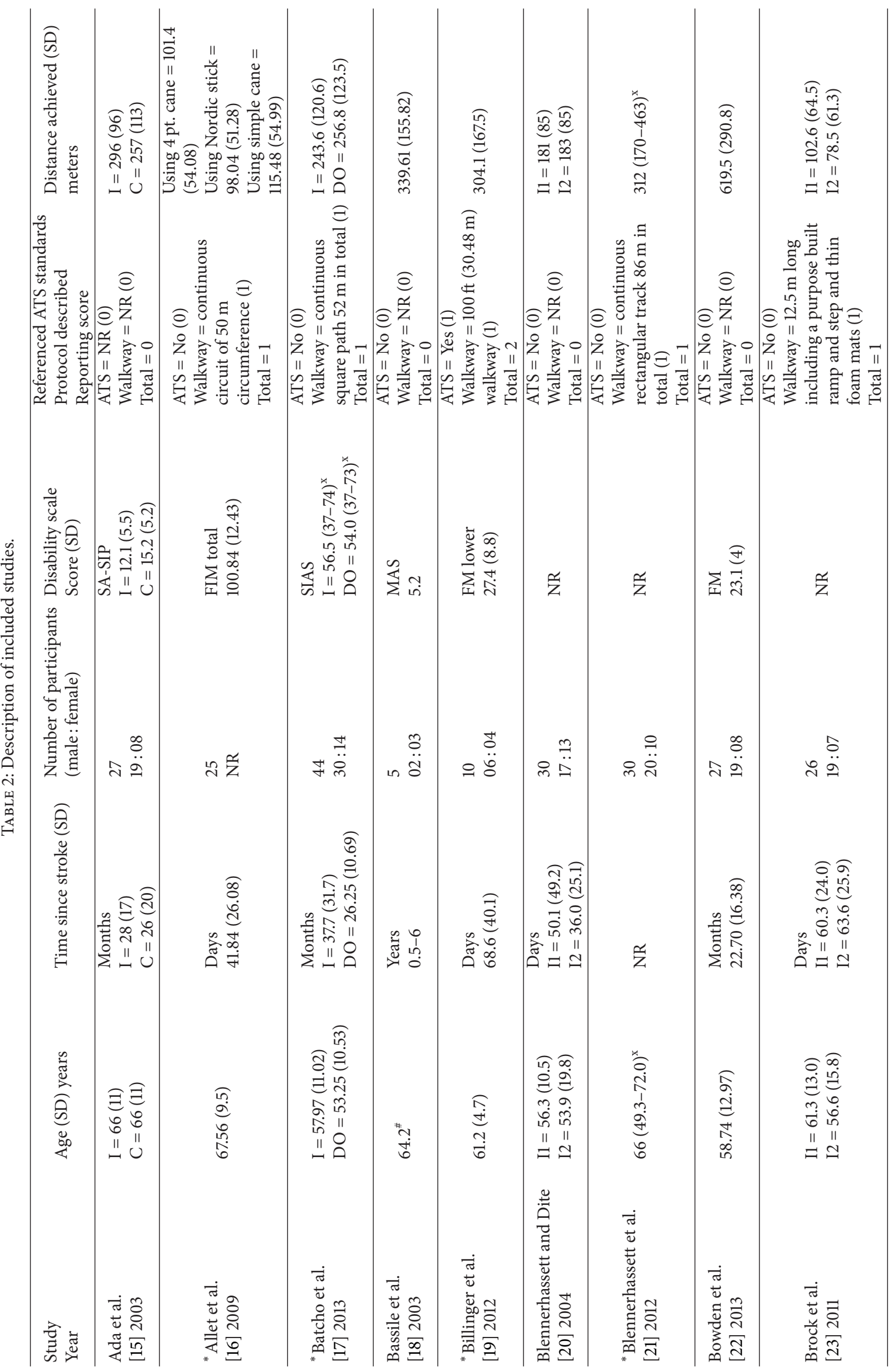




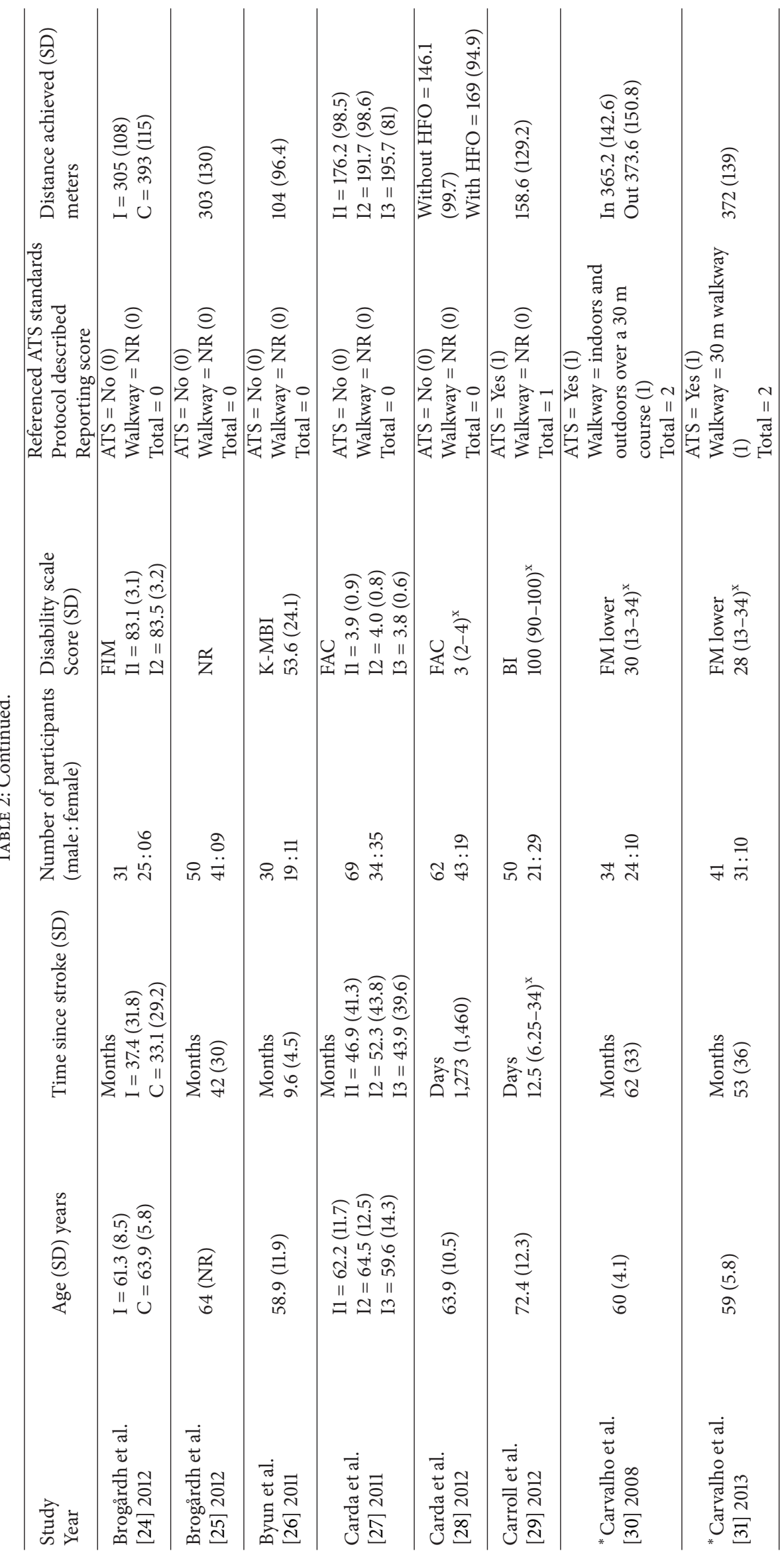




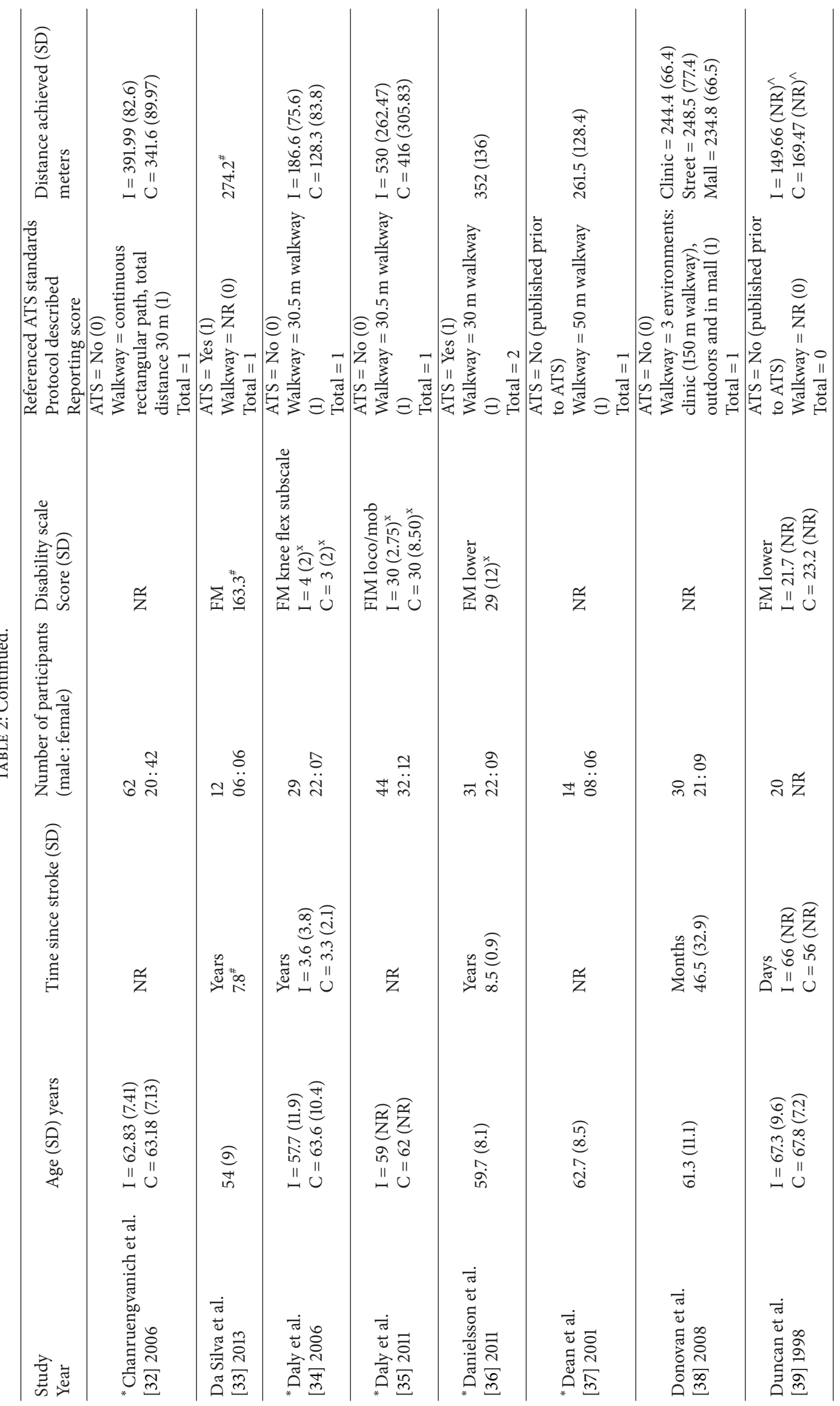




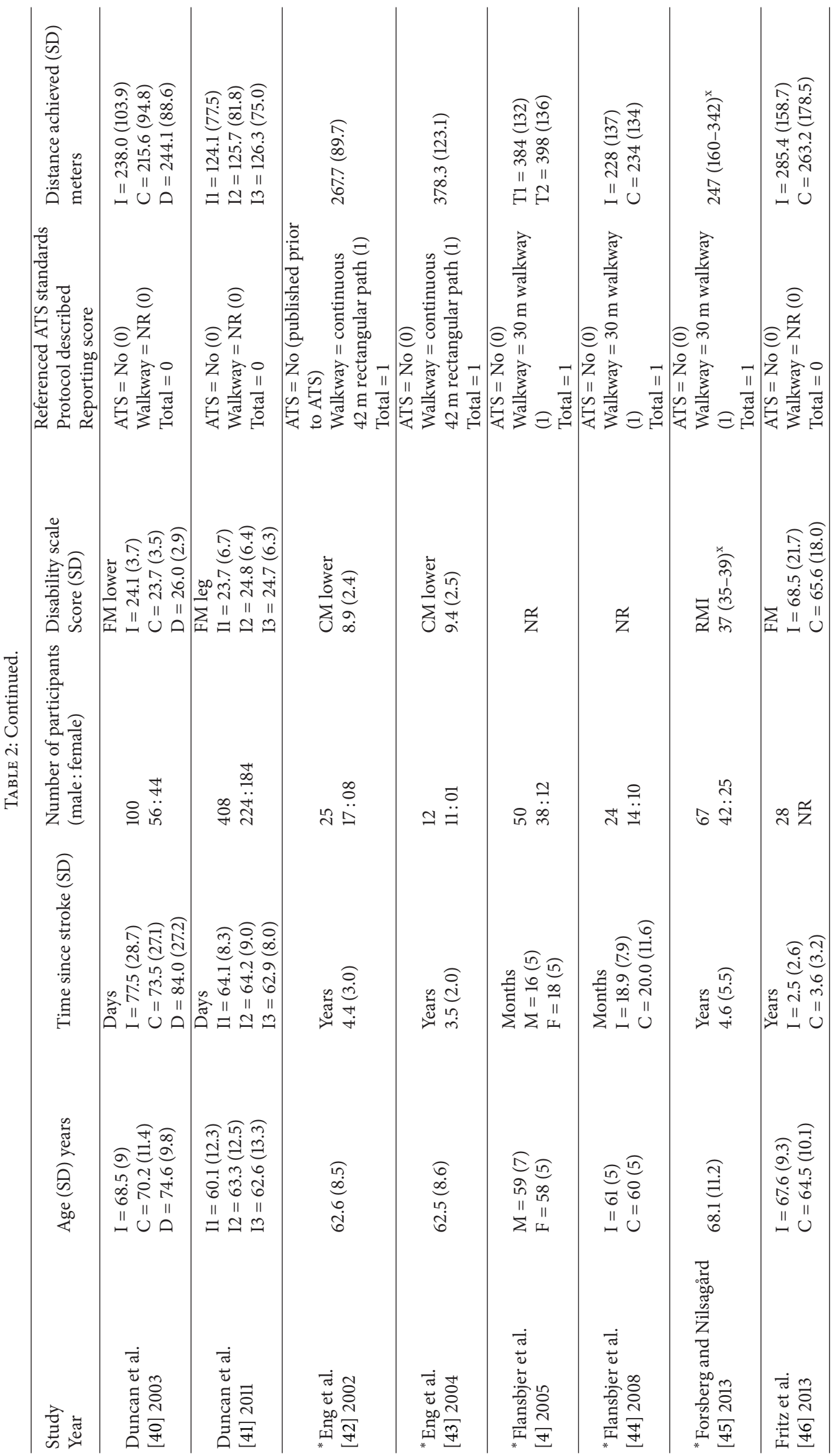




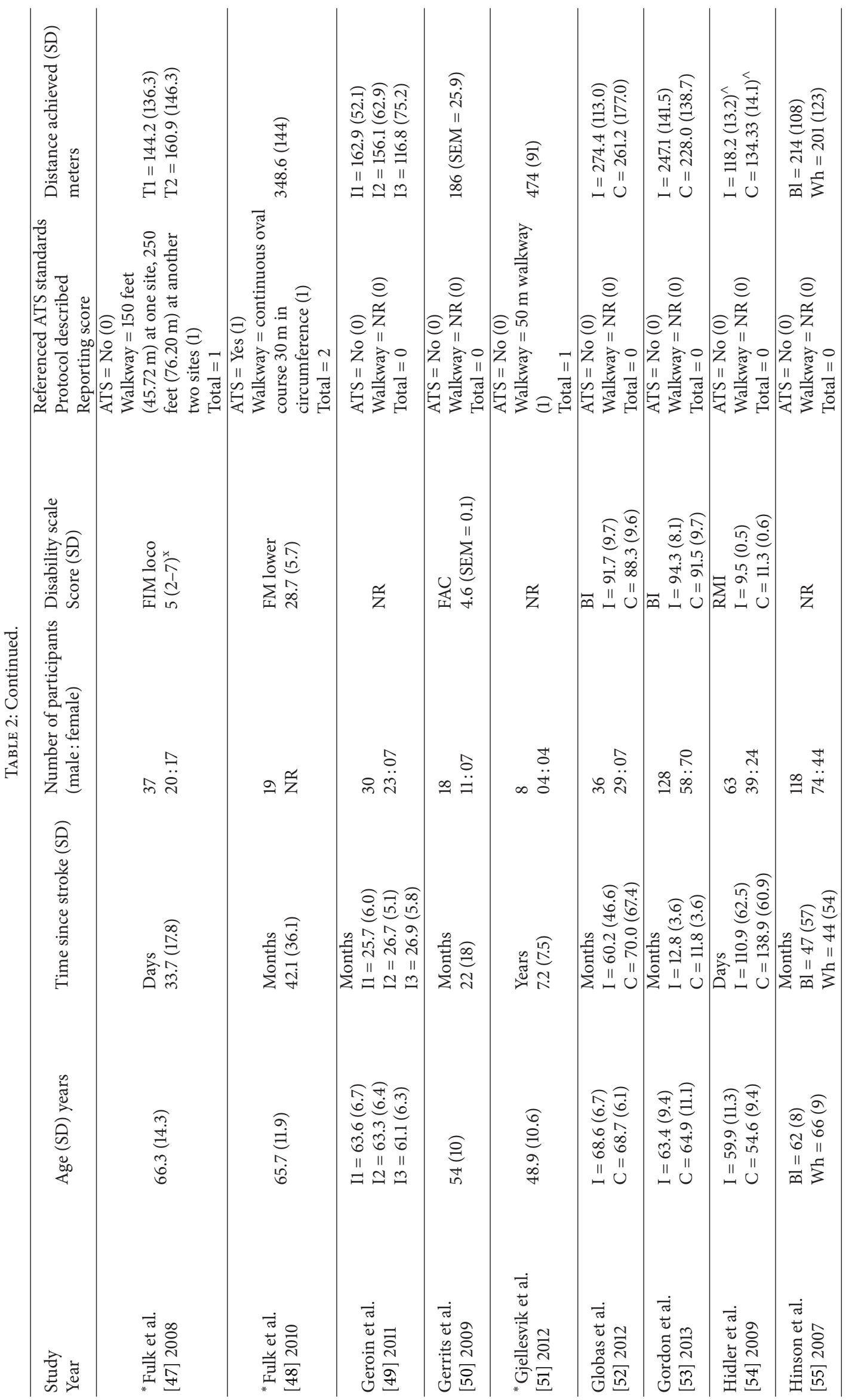




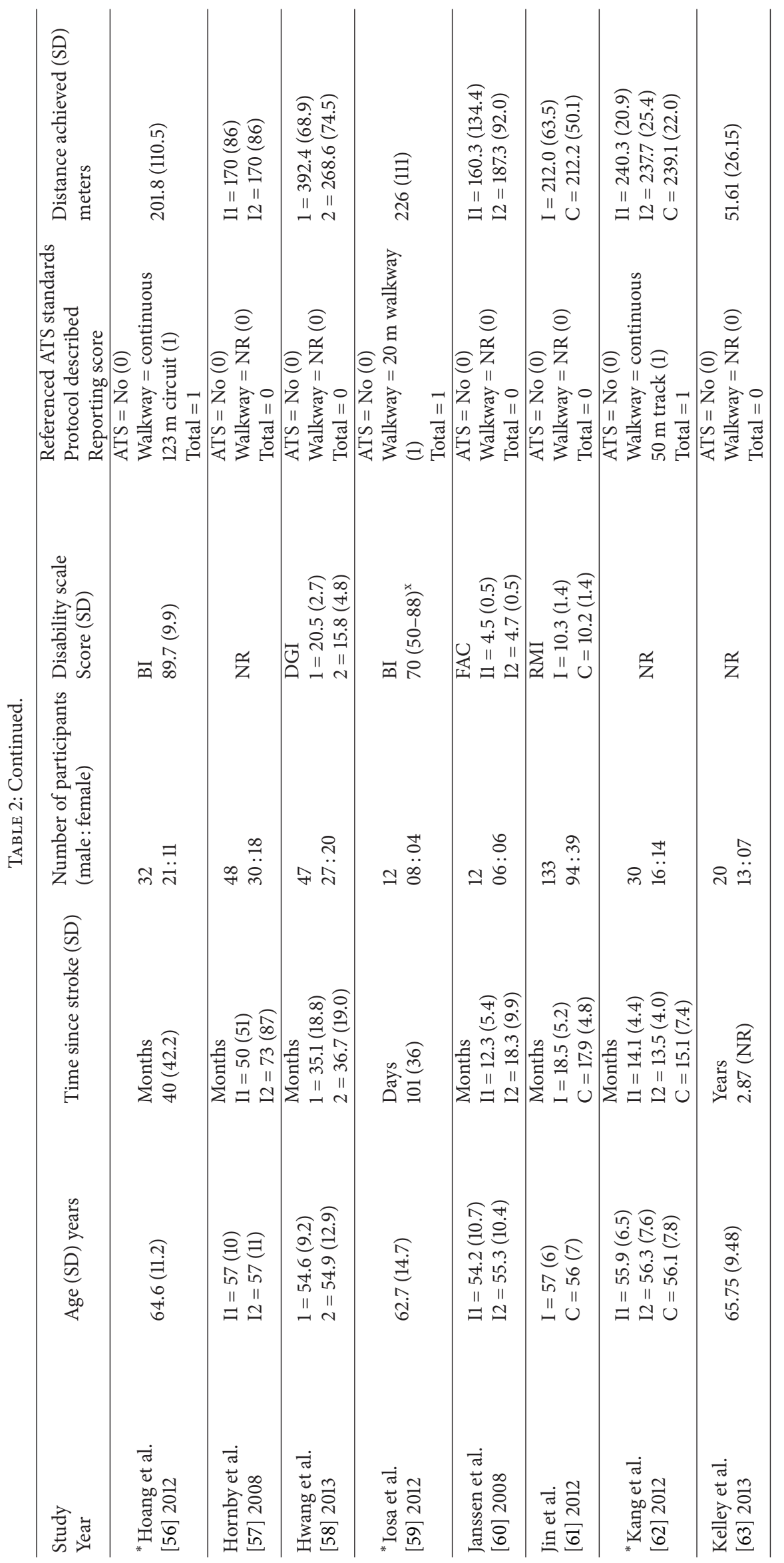




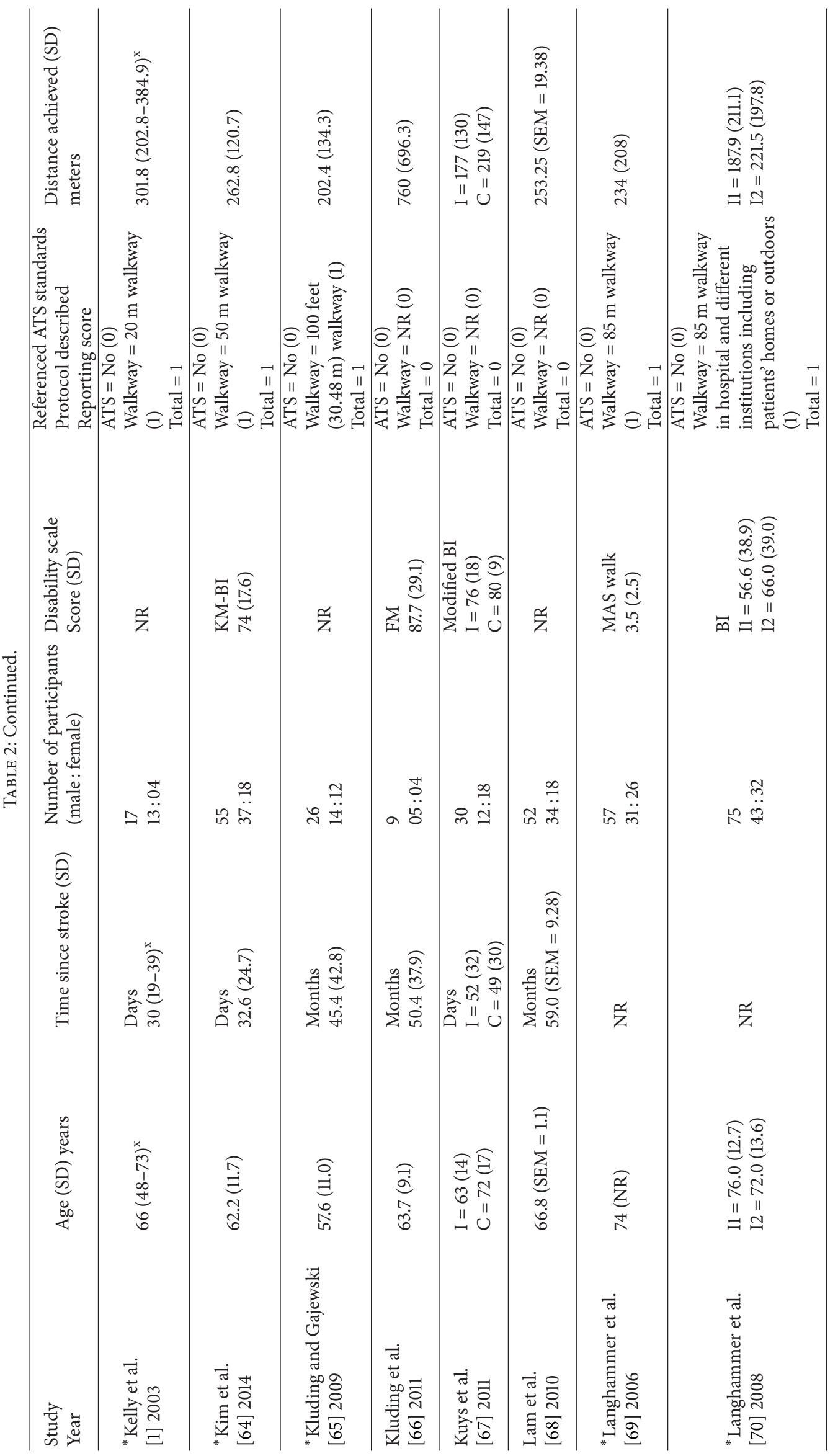




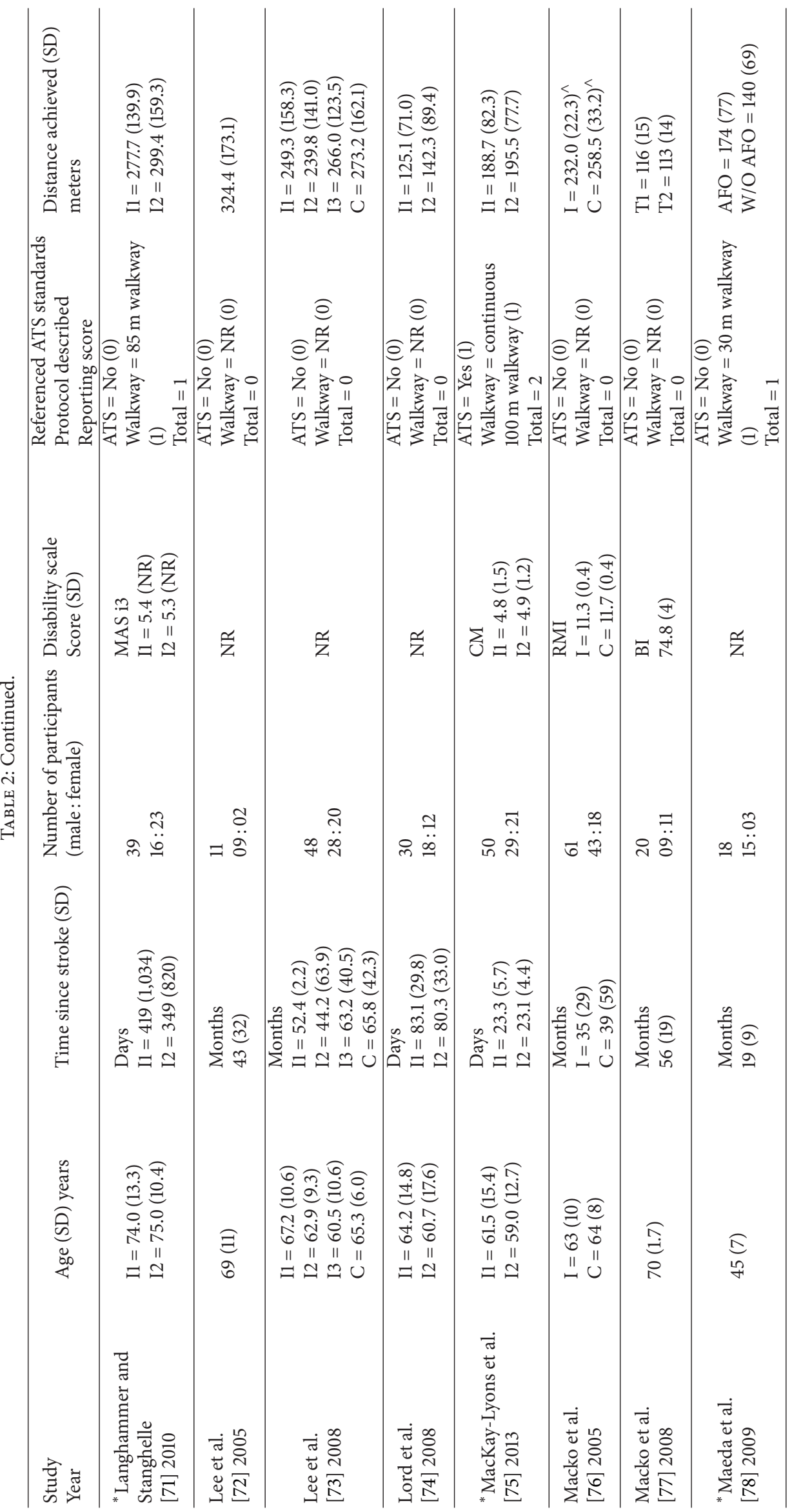




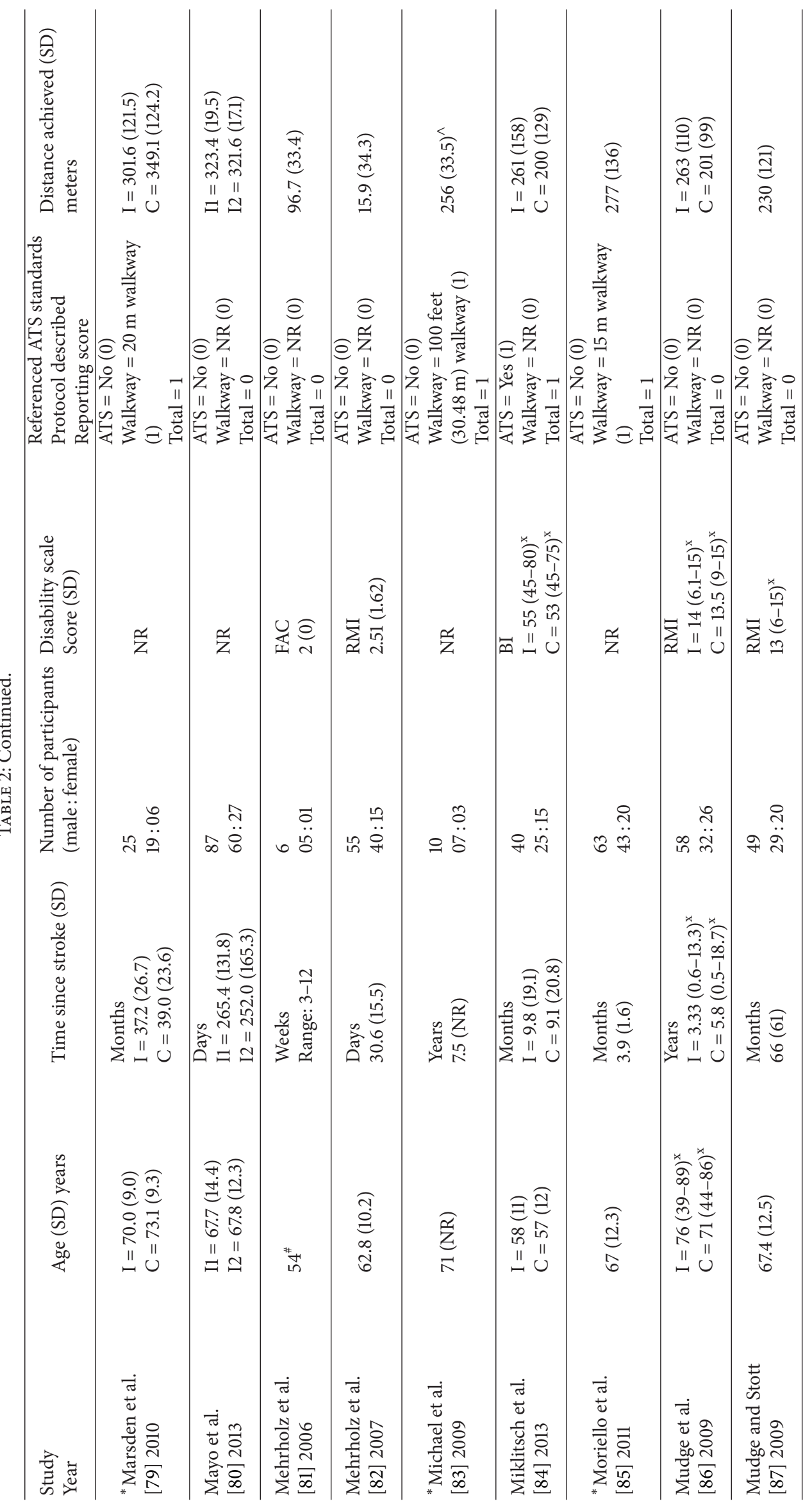




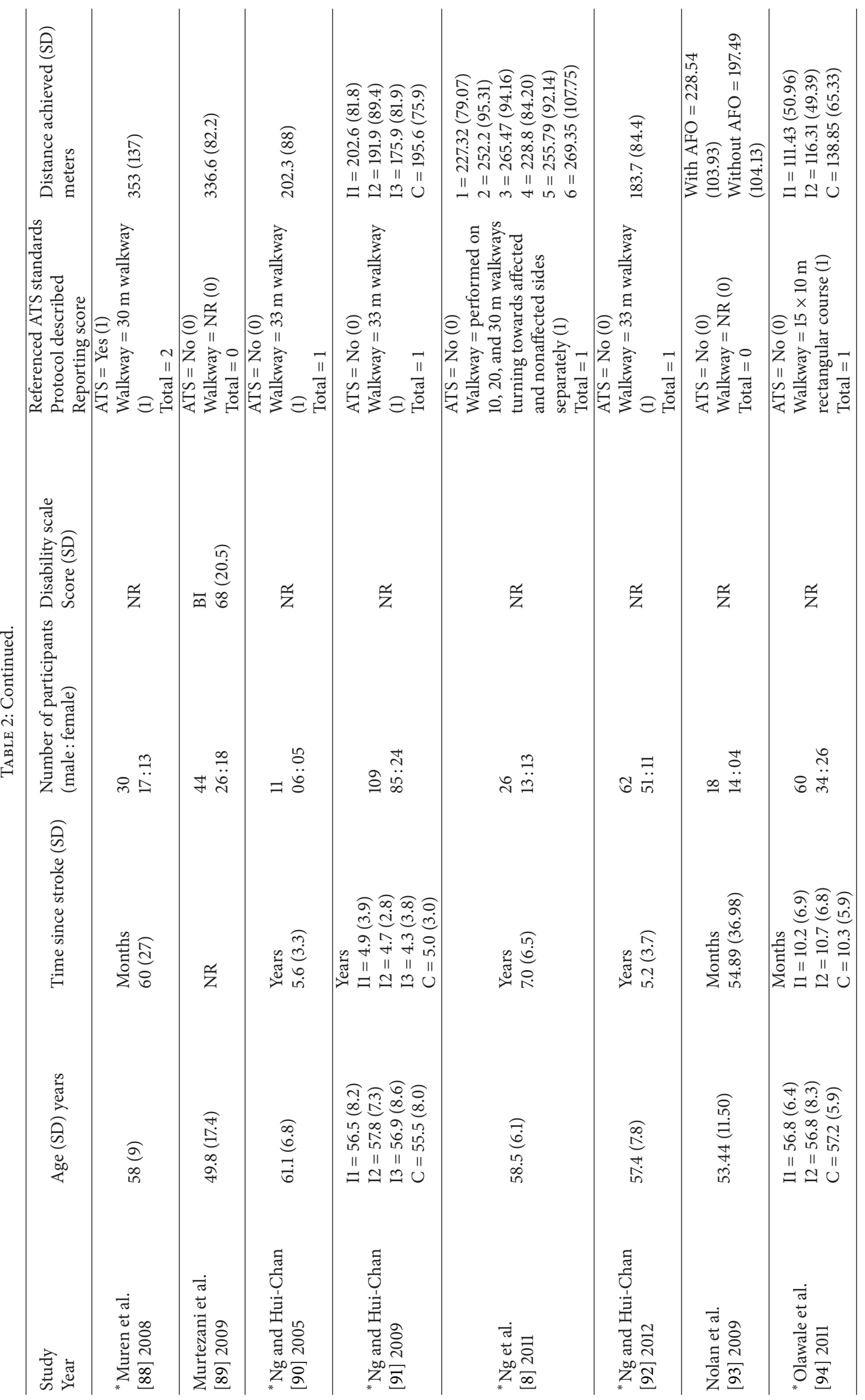




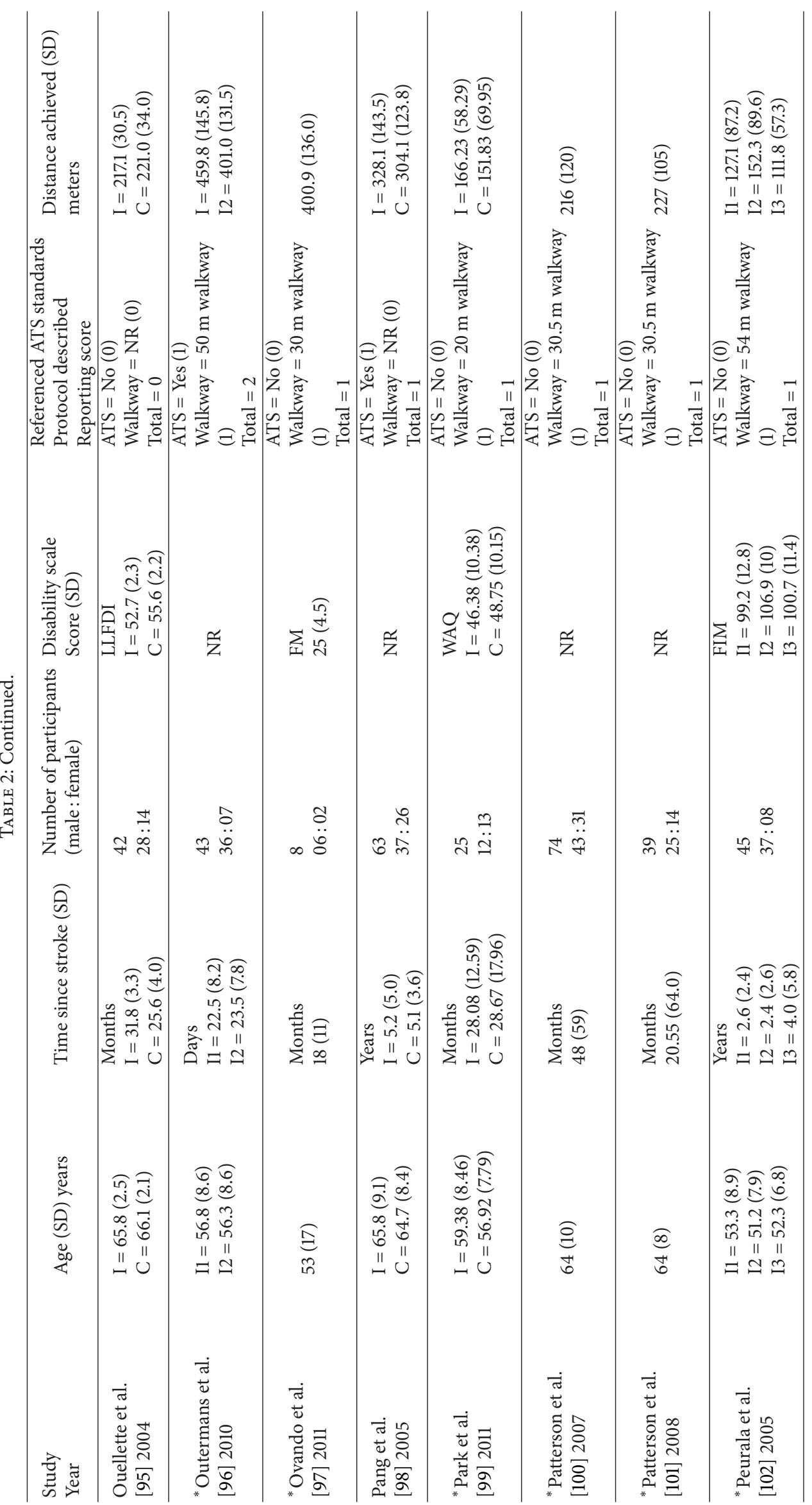




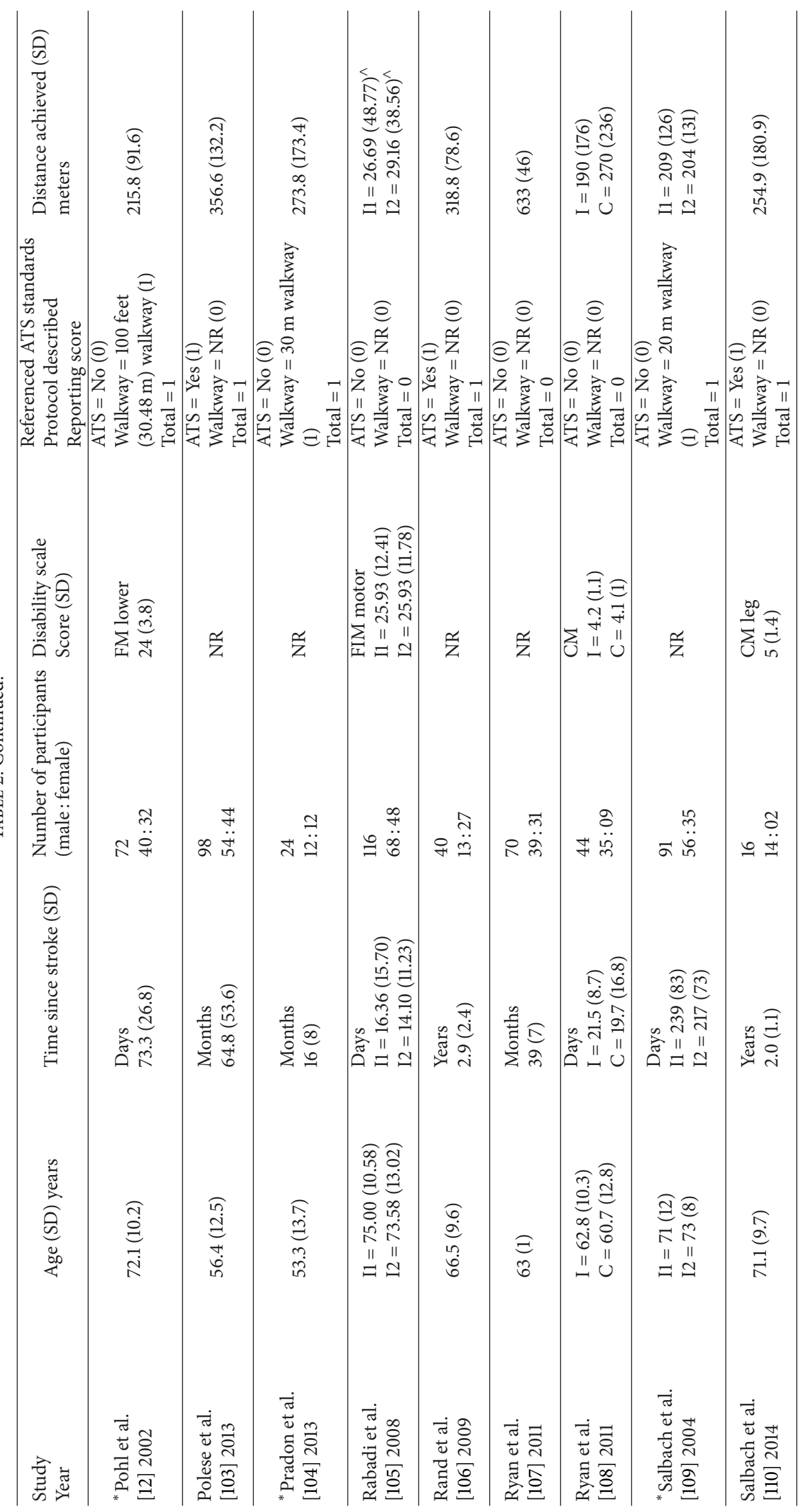




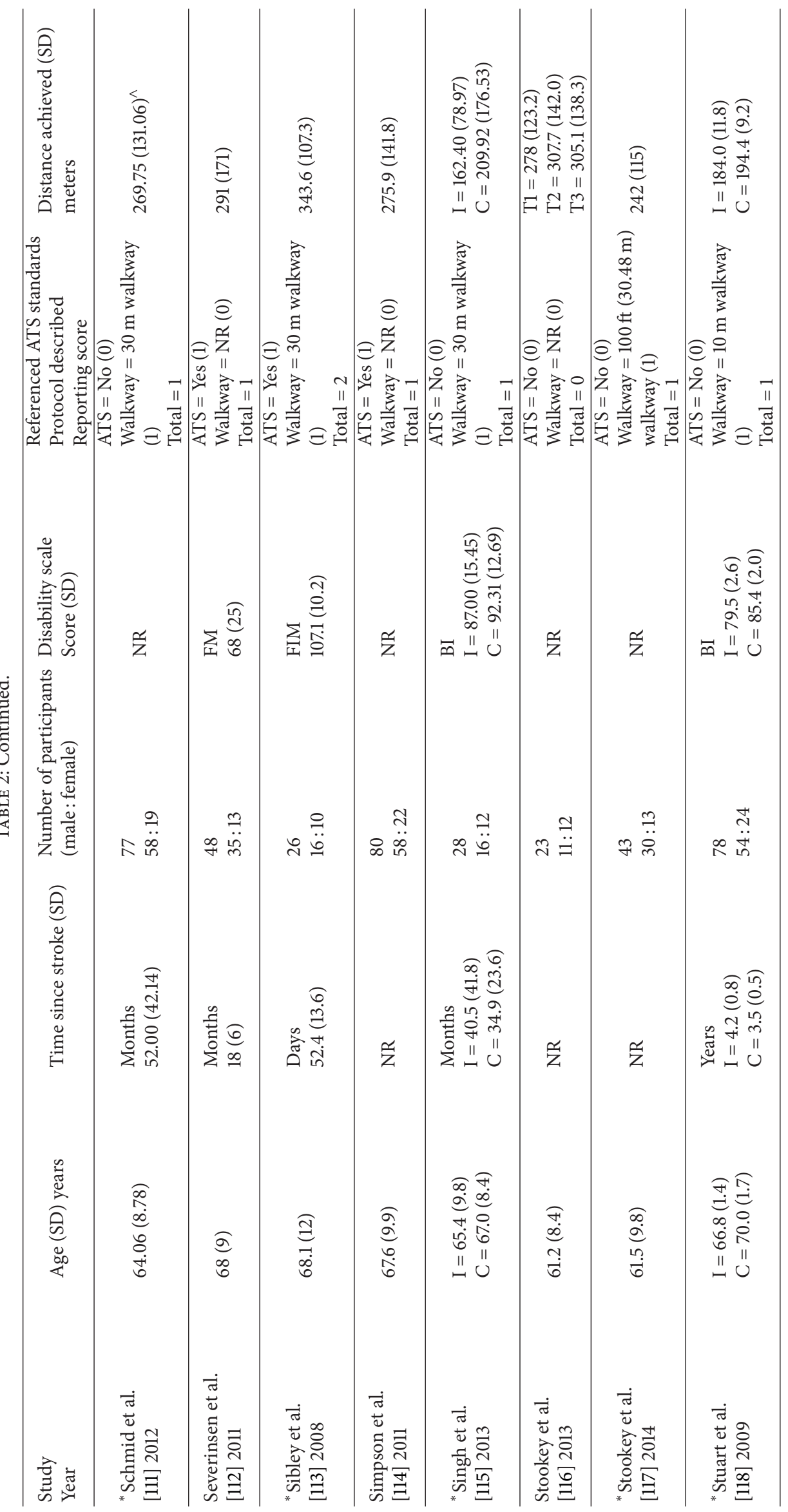




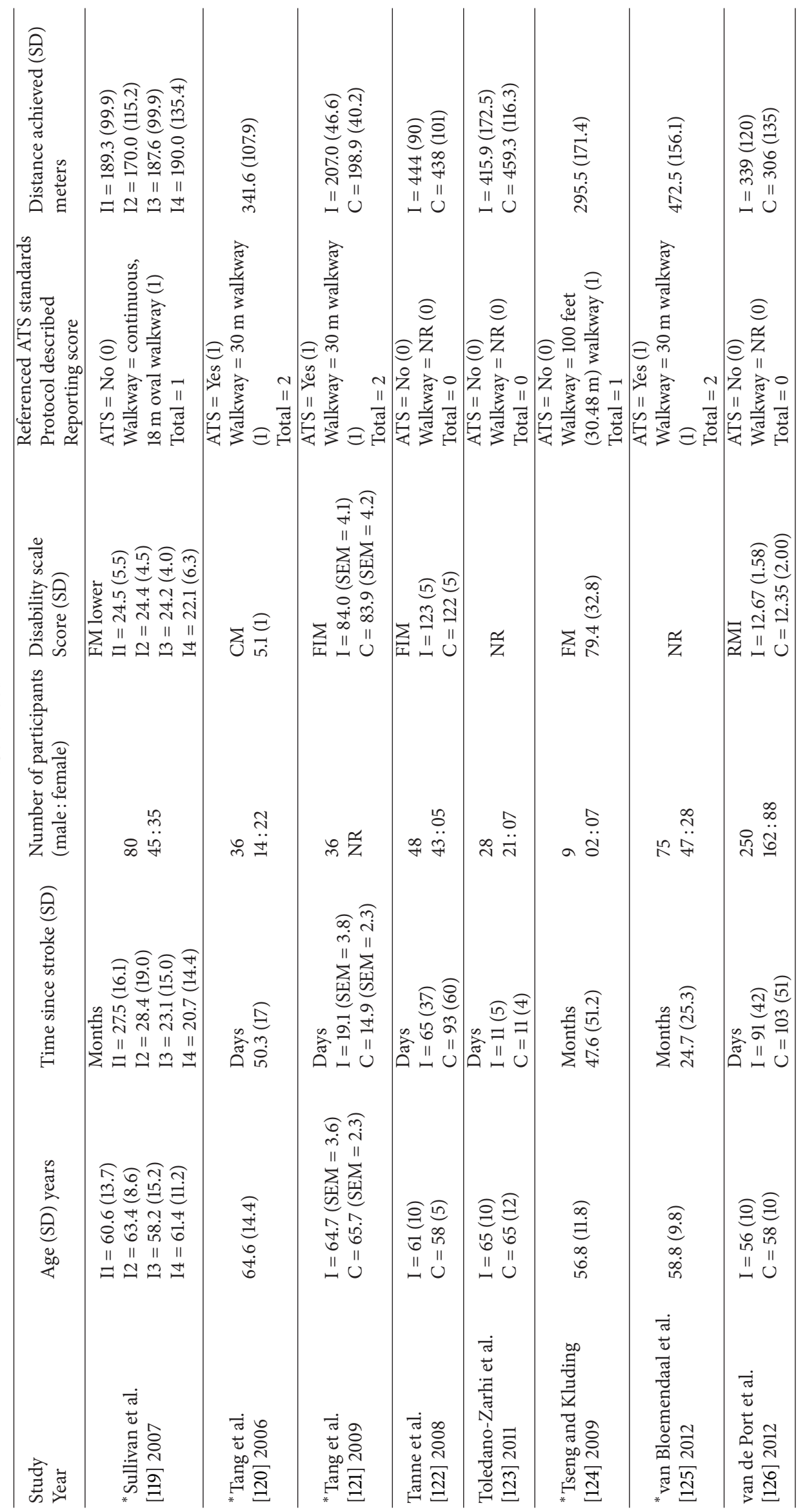




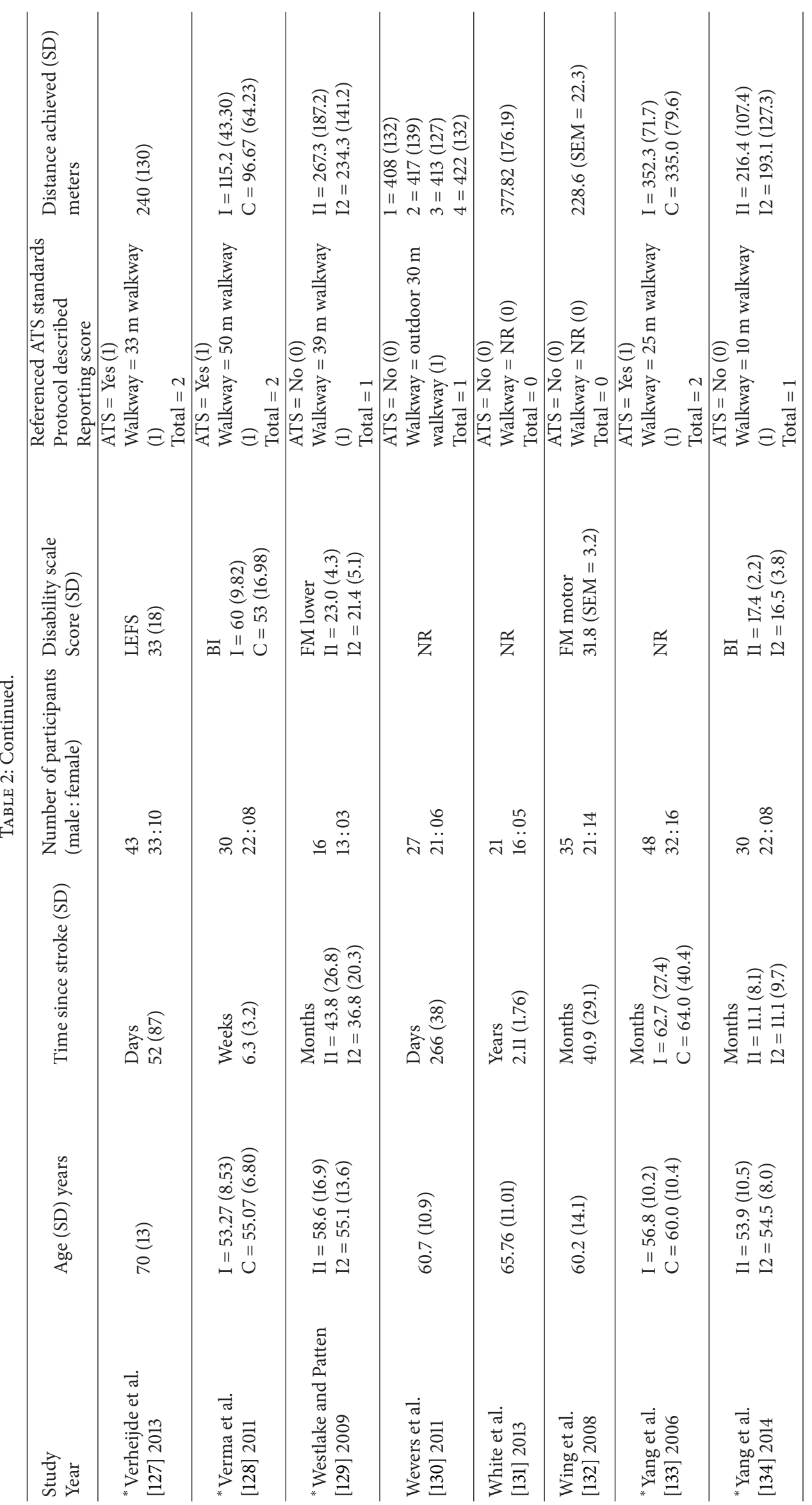




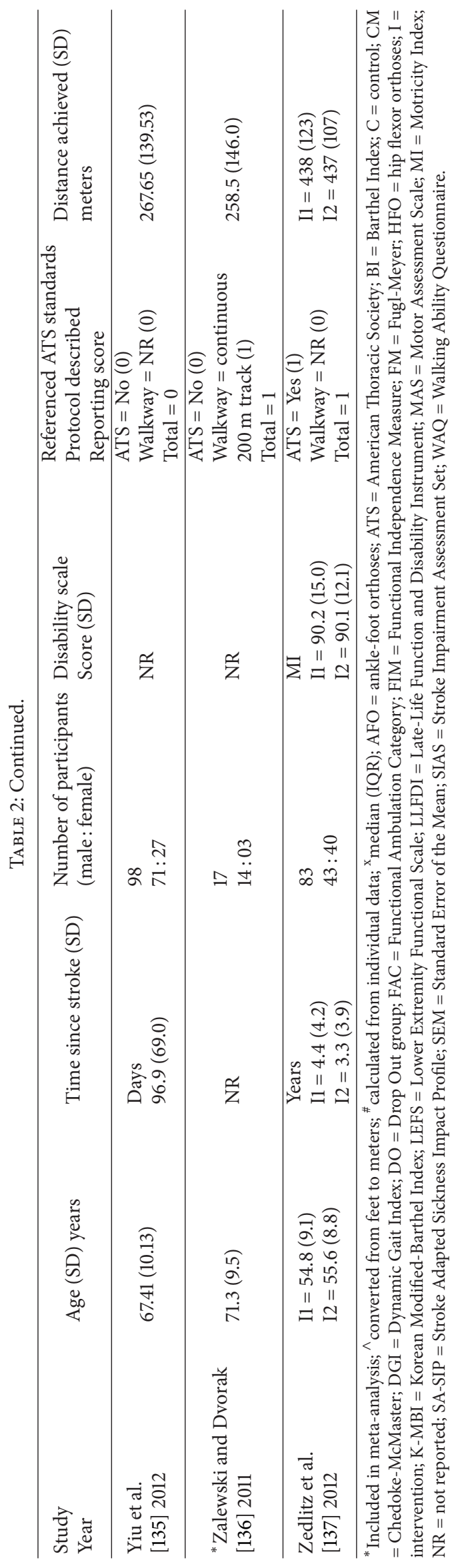


walk on a treadmill as inclusion criteria. These criteria imply the ability to walk, even if it is not explicitly stated.

Participant mean ages (SD) ranged from 45 (7) [78] to 76 (13) [70] years, and mean time since stroke varied from 11 (4) days [123] to $8.5(0.9)$ years [36]. Thirteen studies did not specify time after stroke. Most studies included participants either within 6 months after stroke $(n=32)$, or $1-3$ years $(n=27)$ or more than 3 years after stroke $(n=50)$, with much smaller numbers in the 6-12-month range $(n=9)$. A disability score was reported in only $58 \%$ of all studies. Of those that did report degree of disability, $12 \%$ reported the use of more than one scale. Common disability scales reported in these studies were the Fugl-Meyer Assessment (FM) $(n=24)$, Barthel Index (BI) $(n=13)$, Functional Independence Measure (FIM) $(n=12)$, Rivermead Mobility Index $(n=10)$, Functional Ambulatory Category (FAC) $(n=8)$, Chedoke McMaster $(n=6)$, Motor Assessment Scale (MAS) $(n=6)$, and the Motricity Index (MI) $(n=3)$. Other scales included the Walking Ability Questionnaire $(n=1)$, Dynamic Gait Index (DGI) $(n=1)$, the Late-Life Function and Disability Instrument (LLFDI) $(n=1)$, and the Modified Korean Barthel Index $(n=2)$. Subscale specific lower-limb measures were preferred in the meta-analysis, and these were available in the FM (motricity lower limb, lower extremity) $(n=10)$, FIM (mobility, walking capacity, and locomotion) $(n=7)$, Chedoke McMaster (leg) $(n=5)$, and MAS (walking) $(n=3)$ only.

3.2. Context of the 6MWT. A variety of terms were used to describe the purpose of the 6MWT, with common descriptors being a test of endurance $(n=26)$, capacity $(n=22)$, function $(n=21)$, performance $(n=6)$, and ability $(n=5)$. Twenty-four studies did not report the purpose of conducting the 6MWT in their study. On several occasions the test was performed in a different context or for a different purpose to that described in the ATS guidelines. The 6MWT was performed: to induce fatigue [124]; over a variety of obstacles such as foam mats and purpose built ramps [23]; and in nonstandard locations such as outdoors [30, 130, 138] including suburban streets or shopping centres [38]. Walking distances achieved in outdoor locations ranged from $175 \pm$ $67 \mathrm{~m}$ to $463 \pm 84 \mathrm{~m}$ [36], with participants walking $234 \pm$ $66.5 \mathrm{~m}$ in the shopping centre [127]. Stroke survivors in two intervention groups who walked over foam mats and purpose built ramps [10] walked $102.6 \pm 64.5 \mathrm{~m}$ and $78.5 \pm 61.3 \mathrm{~m}$.

3.3. Assistance and Instructions Provided. Assistance provided to participants during the 6MWT was reported in 24 studies, with most of these studies indicating no assistance or minor assistance required. The single point cane was the most commonly used assistive device $(n=426)$ used during the 6MWT. Other devices used included the walker $(n=106)$, quad cane $(n=77)$, and crutch $(n=5)$. A total of 251 stroke survivors used an ankle-foot orthosis (AFO) during the test. Reporting use of a "usual device" without specifying the device used was prevalent ( $n=310$ participants).

Only $44 \%$ of studies reported the instructions provided to participants for the $6 \mathrm{MWT}$ with variations evident between studies. The most common phrase used was to "cover as much distance as possible" ( $n=20$ studies), followed by "walk as far as you can" $(n=16)$. Five studies specified walking at a fast pace in their instructions, and eight studies instructed participants to walk at a comfortable speed. Encouragement was provided in 10 studies, and no encouragement or verbal feedback was given in 14 studies.

3.4. Quantitative Analysis of Adherence to ATS Protocol Guidelines. Including the three studies published prior to the ATS standards, 49 of the 127 studies received a zero score, indicating that they did not mention the ATS guidelines and did not describe a protocol for the test including walkway length or course design. Sixty-three studies received a score of one indicating that these studies either referenced the ATS standard or provided details on the walkway length used. Of these, 53 received one point for reporting the walkway length or course design, while only ten received one point for referencing the ATS guidelines. Only 15 studies scored two points, with only nine of these reporting a reference to the ATS guidelines and complying with them by using a $30 \mathrm{~m}$ walkway.

3.5. Modifications to the 6MWT Protocol Walkway Length. Only 25 of the 127 studies made a reference to the ATS guidelines for the 6MWT, with only nine of these clearly reporting the use of a $30 \mathrm{~m}$ walkway. Although referencing the ATS standards, six studies modified the protocol with variations including the use of a $25 \mathrm{~m}$ walkway [133], a $33 \mathrm{~m}$ walkway [127], a $50 \mathrm{~m}$ walkway [96, 128], a $100 \mathrm{~m}$ walkway [75], and a $30 \mathrm{~m}$ oval course [48]. Overall, 67 studies provided a description of the walkway whereas 60 studies did not provide any description of the length or shape of the walkway used. Of those providing a description, 27 reported using an indoor $30 \mathrm{~m}$ walkway in accordance with the guidelines while 10 used shorter walkway lengths, 14 used longer walkways lengths, and 14 used continuous walkways. Straight walkway lengths varied from $10 \mathrm{~m}$ [118] to $85 \mathrm{~m}$ [69-71].

3.6. Distances Achieved Using the 6MWT in Stroke Survivors. Sixty-four studies were included in the meta-analysis. The pooled distance walked across these 64 studies was $247.3 \mathrm{~m}$ $(\mathrm{SE}=9.09)$. Heterogeneity was high with a tau (tau squared represents between study variance) of $84.9 \mathrm{~m}$. We explored whether this heterogeneity was due to track type. Stroke survivors achieved a distance of $285 \mathrm{~m}$ (95\% CI, 252-318 m) on a $30 \mathrm{~m}$ track. A significantly greater distance was achieved using the $30 \mathrm{~m}$ walkway compared to protocols with longer (231 $\mathrm{m}, 95 \%$ CI 189, 272, $P=0.048)$ or continuous $(213 \mathrm{~m}$, $95 \%$ CI 171, 255, $P=0.010$ ) walkways (Figure 2); there was no significant difference between the $30 \mathrm{~m}$ walkway and shorter ( $242 \mathrm{~m} 95 \%$ CI 199, 286, $P=0.122$ ) walkway lengths. Of the 60 studies that did not provide a description of the walkway used, an average distance of $246 \pm 117 \mathrm{~m}$ was reported. Differences in distributions of age, gender, or time since stroke did not have a significant influence on distance walked (Table 3). Disability scores were only available on 74 (58\%) studies and therefore could not be included in the analysis. 
TABLE 3: Meta-regression coefficients for all studies.

\begin{tabular}{lccccccc}
\hline Effect & Estimate & Std. Err. & DF & $t$ value & Probt & Lower CI & Upper CI \\
\hline Age & 0.6 & 1.7 & 75 & 0.38 & 0.708 & -2.7 & 4.0 \\
Proportion males & 0.6 & 0.8 & 75 & 0.75 & 0.455 & -1.0 & 2.2 \\
Time since stroke & 0.5 & 0.4 & 75 & 1.16 & 0.251 & -0.3 & 1.3 \\
\hline
\end{tabular}

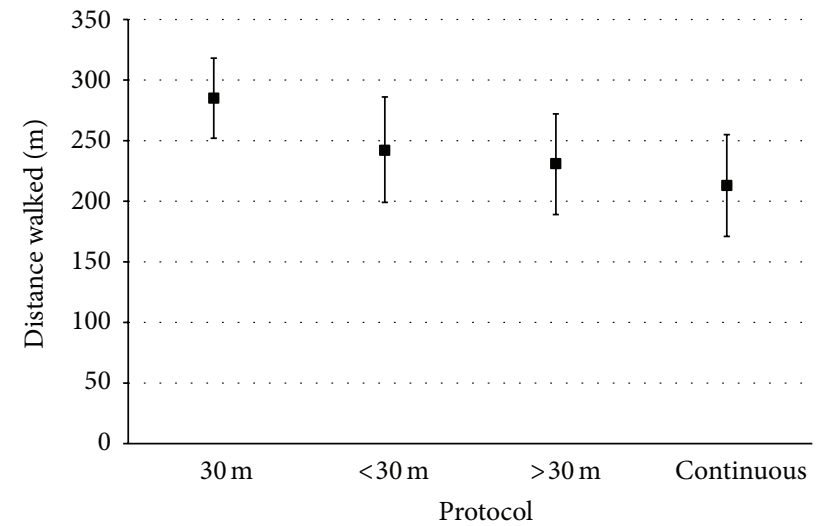

FIGURE 2: Distances achieved (point estimate, 95\% CI) during the $6 \mathrm{MWT}$ based on walkway protocol used.

The regression analysis conducted in the 46 studies reporting use of the $30 \mathrm{~m}$ walkway found that none of the variables, that is, age $(P=0.479)$, gender $(P=0.768)$, or time after stroke $(P=0.909)$, were significantly associated with distance achieved on the 6MWT in stroke survivors (Table 4). Disability scores were only available on $12(12 / 46,26 \%)$ of these studies and therefore were not included in this analysis.

\section{Discussion}

This review has identified that the 6MWT is now in widespread use to assess aspects of walking-related performance in stroke survivor studies. Many of the study populations had to meet strict eligibility criteria including being able to meet a minimal walking speed and no comorbidities and therefore may only be selectively representative of stroke survivors. Many stroke survivors would not be able to meet the criteria for these studies, making a bias towards more well recovered, nondisabled, otherwise healthier participants. However, even in this selected population, the distances achieved on the 6MWT indicate substantial walking limitations in people after stroke. Overall, both the reporting of and adherence to the ATS guidelines for the 6MWT regarding the walkway length could be improved. Similarly, reporting of the instructions given prior to testing, as well as any assistance provided to participants during the test, requires attention in future publications. Alterations to the ATS protocol walkway, including shortening or extending the walkway length or using a continuous track, are more common than adhering to the $30 \mathrm{~m}$ walkway length. Consequently, a set of guidelines has been developed for future reporting of the 6MWT (Table 5). The findings from the meta-analysis were that the distance achieved during the 6MWT was associated with variations to the walkway length, but not in the manner one would predict. These findings have implications for the comparison of the values achieved using the $6 \mathrm{MWT}$ in different studies of stroke survivors.

The introduction of the ATS guidelines in 2002 was aimed at providing a protocol for consistency between studies. Of the 127 studies included in this review, 39 described a modified protocol whereas 60 studies did not specify the walkway length or walking course design. Of the 15 studies that received a reporting score of two, only nine reported both the ATS guidelines and used a $30 \mathrm{~m}$ walkway length in accordance with the guidelines: the remaining six studies referenced the ATS guidelines but reported using a modified track. Consequently, although the ATS guidelines may be referenced in a report, it cannot be assumed that there was adherence to the guidelines. Protocol modification was more common than compliance with the $30 \mathrm{~m}$ ATS standards, but no studies reported a reason for changing the protocol. Although there may be factors that necessitate or justify protocol changes, these reasons remain unreported. It is understandable that, in a setting where space is limited, there may be no other option than to use a walkway distance of less than $30 \mathrm{~m}$. Reasons for lengthening the walkway are less clear. One explanation is that if space greater than $30 \mathrm{~m}$ in length is available, then a reason to extend the walkway length above the standard would be to decrease the effects of turning for stroke survivors in whom turning and balance ability may be compromised. We anticipated that the reduced turning requirements on the extended walkways and continuous tracks would result in longer distances being achieved than on the standard $30 \mathrm{~m}$ walkway. The results of the meta-analysis show the opposite, with these protocols resulting in shorter 6MWT distances. One possible explanation for these types of protocol changes would be to accommodate reduced turning abilities and more severe disability of participants in these studies, which may also explain the reduced walk distances achieved.

The main impact of varying the 6MWT track length or design is the extent of turning required throughout the test. Turning requires the integration of multiple sensory systems and utilises vestibular, visual, and proprioceptive information to appropriately move the body in space. Stroke survivors often experience difficulty during turning, possibly as a result of altered sensory, motor, and biomechanical systems [139]. This results in a differing orientation and sequencing of movements during turning compared to healthy controls [5], which requires more time to complete. Stroke survivors may take almost twice as many steps and twice as much time to complete a $180^{\circ}$ turn compared to age-matched controls [140]. Similarly, two studies have reported significantly slower $180^{\circ}$ 
TABle 4: Meta-regression coefficients for $30 \mathrm{~m}$ protocol subgroup.

\begin{tabular}{lccccccc}
\hline Effect & Estimate & Std. Err. & DF & $t$ value & Probt & Lower CI & Upper CI \\
\hline Age & -2.4 & 3.3 & 25 & -0.72 & 0.479 & -9.1 & 4.4 \\
Proportion males & 0.4 & 1.4 & 25 & 0.30 & 0.768 & -2.5 & 3.3 \\
Time since stroke & -0.1 & 0.7 & 25 & -0.12 & 0.909 & -1.5 & 1.4 \\
\hline
\end{tabular}

TABLE 5: Checklist for reporting of the 6MWT.

\begin{tabular}{l}
\hline Checklist for reporting of the 6MWT \\
(1) Check \\
(a) Acknowledge awareness of the ATS standards by referencing \\
(b) Report walkway length even if it is $30 \mathrm{~m}$ \\
(c) If deviating from ATS standards, describe changes (walkway length, course layout, and location) and explain reason \\
$\begin{array}{l}\text { (2) } \\
\text { (a) Describe the instructions given prior to the test }\end{array}$ \\
(b) Describe any encouragement provided during the test \\
\hline (3) Report the number and type of assistive devices used \\
\hline (4) Report any assistance or support provided to participants \\
\hline (5) \\
(a) Report the demographics of the population including disability level \\
(b) Report clearly the inclusion and exclusion criteria \\
\hline
\end{tabular}

turning times during the Timed Up and Go (TUG) in stroke $[10,141]$ with a similar finding by van Herk et al. (1998) [5] who demonstrated that the time taken to walk $10 \mathrm{~m}$ straight versus $5 \mathrm{~m}$ with return in stroke survivors was different, with the $5 \mathrm{~m}$ track with return requiring significantly more time to complete $(P<0.001)$. When comparing stroke survivors' performances on the $6 \mathrm{MWT}$ over 10, 20, and $30 \mathrm{~m}$ tracks, $\mathrm{Ng}$ et al. (2011) [8] quantified the increased number of turns associated with shortening the track and reported a significantly shorter distance achieved during the $10 \mathrm{~m}$ protocol, with the $30 \mathrm{~m}$ protocol reporting the longest distance walked. This is contradictory to the findings in this review; however when looking at percent difference there is some commonality. In the study by $\mathrm{Ng}$ et al., there is a reported reduction in distances achieved of $5 \%(20 \mathrm{~m}$ compared to $30 \mathrm{~m})$ to $15 \%(10 \mathrm{~m}$ compared to $30 \mathrm{~m})$. The results from the current meta-analysis suggest a reduction of $15 \%$ when comparing the $<30 \mathrm{~m}$ protocol to the $30 \mathrm{~m}$ walkway length. Although this was not statistically significant, it is consistent with the magnitude of compromise found in the study by $\mathrm{Ng}$ et al. who measured the same population over multiple walkway lengths and could therefore make a direct comparison.

Another important finding from $\mathrm{Ng}$ et al. was that turning direction did not influence 6MWT distance independent of walkway length. The effects of turning direction on TUG times were also investigated in two other studies. Faria et al. [141] also found no difference in TUG times. In contrast Heung and $\mathrm{Ng}$ [142] found a significantly faster TUG time when turning towards the paretic side. There appears to be an expectation that turning towards the affected side would result in slower speeds and therefore decreased 6MWT distance; however as demonstrated in the literature, this may not be the case. Generally, the studies in this review did not specify the directions turned and therefore could not be analysed. It is noted however that in the clinical setting it is usually at the discretion of the individual performing the test as to which direction they turn during the 6MWT.

Our findings highlight the substantial effects of stroke on walking speed. Stroke survivors achieved an average distance of $285 \mathrm{~m}$ (95\% CI 252, 318) on a standard $30 \mathrm{~m}$ track, whereas healthy older individuals $>60$ years achieve an average 6MWT distance of $499 \mathrm{~m}$ (95\% CI 480, 519) [143]. The extent of the performance compromise on the 6MWT is striking, particularly when the studies in this review are largely reporting on a highly selected, high performing cohort of independently ambulant stroke survivors. When considering these distances achieved, it is important to also acknowledge any assistive devices used throughout the 6MWT. Overall, 948 stroke survivors used a walking aid, and 251 walked with an AFO. Allet et al. [16] found that stroke survivors walked approximately $15 \mathrm{~m}$ further during the 6MWT using a simple cane with an ergonomic handgrip than when walking with a 4-point cane or Nordic stick. This area requires further investigation and should be considered when interpreting data.

Of the variables age, gender, and time after stroke, none had a significant effect on the distances achieved. This is likely because meta-regression was performed using summary level data for each study, rather than individual patient data that would have more power to tease out heterogeneity. In healthy adults, the variables age and gender have been suggested as sources of variability on distances achieved [9].

Unfortunately, the effect of disability on distance walked could not be discerned in this review due to the large number of different disability scales used to describe stroke 
populations, the lack of consistency of scales between studies, and the underreporting of mobility related disability. An attempt to use an alternative measure of motor function, such as $10 \mathrm{~m}$ walking speed or balance, was also unsuccessful, as few of the studies in the meta-analysis reported on these measures.

The instructions provided to stroke survivors differ between studies, potentially impacting on the perceived goal of the 6MWT. In the current analysis, five studies reported wording to walk at a "fast" pace, while nine studies instructed participants to walk at a "comfortable" pace. According to the ATS guidelines, instructions should be informing the participant to walk "as far as possible for 6 minutes" with no mention of walking speed. The guidelines also provide standard encouragement wording to use. Of those studies that reported on the encouragement given throughout the test, $42 \%$ provided encouragement and $58 \%$ provided no verbal encouragement or feedback. The guidelines specify that the 6MWT should be performed indoors; however several studies reported using the 6MWT in different environments such as outdoors $[36,124,126]$ including suburban streets and in shopping centres [127]. Although these trials were excluded from the meta-analysis, the exact implications of performing the 6MWT in different locations are unclear. Carvalho et al. [138] directly compared distanced achieved indoors and outdoors, concluding that stroke survivors in Group B (self-selected walking speed $\geq 0.8 \mathrm{~m} \cdot \mathrm{s}^{-1}$ ) achieved a greater distance in the outdoor setting, whereas for those in Group A (self-selected walking speed $<0.8 \mathrm{~m} \cdot \mathrm{s}^{-1}$ ) there was no difference. It has also been suggested that gait parameters do not differ in stroke survivors when walking in different environments [127]. This is another factor that should be considered when interpreting the results reported from the 6MWT.

This review highlights the need for future researchers to be mindful in reporting their implementation of the $6 \mathrm{MWT}$ by describing the walkway used and rationales for deviations from the $30 \mathrm{~m}$ walkway or other aspects of the protocol. To assist future researchers we have developed a checklist of items for unambiguous reporting of the use of the 6MWT (Table 5). We acknowledge that changing the protocol may be necessary in some settings due to space restrictions. If the primary purpose of the test is to compare performances prepost intervention then consistency is the main requirement between tests. Each setting may require their own documented protocol in order to maintain consistency between measurement times and test supervisors. The effects of level of disability on 6MWT performance could not be readily discerned due to the large number of different disability scales used, with many of these providing little indication of mobility impairment. Similarly, the extent of assistance provided, as well as the instructions given to the participants, requires attention in future studies. It can be concluded from the review that by changing the protocol researchers are limiting the ability to compare results between studies in stroke as well as other clinical populations. There is a lack of comprehensive reporting of the 6MWT protocol, which needs to be addressed in future research publications.

\section{Conflict of Interests}

D. L. Marsden was an author on paper [79], which was an included paper in the review.

\section{Acknowledgments}

The authors would like to acknowledge Debbie Booth (Faculty of Health and Medicine librarian, University of Newcastle) for her assistance with the search, and Dr. Christopher Oldmeadow (Clinical Research Design, Information Technology (CReDITSS), Hunter Medical Research Institute) for his statistical support.

\section{References}

[1] J. O. Kelly, S. L. Kilbreath, G. M. Davis, B. Zeman, and J. Raymond, "Cardiorespiratory fitness and walking ability in subacute stroke patients," Archives of Physical Medicine and Rehabilitation, vol. 84, no. 12, pp. 1780-1785, 2003.

[2] D. L. Marsden, A. Dunn, R. Callister, C. R. Levi, and N. J. Spratt, "Characteristics of exercise training interventions to improve cardiorespiratory fitness after stroke: a systematic review with meta-analysis," Neurorehabilitation and Neural Repair, vol. 27, no. 9, pp. 775-788, 2013.

[3] K. M. Michael, J. K. Allen, and R. F. Macko, "Reduced ambulatory activity after stroke: the role of balance, gait, and cardiovascular fitness," Archives of Physical Medicine and Rehabilitation, vol. 86, no. 8, pp. 1552-1556, 2005.

[4] U. B. Flansbjer, A. M. Holmbäck, D. Downham, C. Patten, and J. Lexell, "Reliability of gait performance tests in men and women with hemiparesis after stroke," Journal of Rehabilitation Medicine, vol. 37, no. 2, pp. 75-82, 2005.

[5] I. E. H. van Herk, J. H. Arendzen, and P. Rispens, "Ten-metre walk, with or without a turn?" Clinical Rehabilitation, vol. 12, no. 1, pp. 30-35, 1998.

[6] R. J. A. Butland, J. Pang, E. R. Gross, A. A. Woodcock, and D. M. Geddes, "Two-, six-, and 12-minute walking tests in respiratory disease," British Medical Journal, vol. 284, no. 6329, pp. 16071608, 1982.

[7] C. R. McGavin, S. P. Gupta, and G. J. R. McHardy, “Twelve minute walking test for assessing disability in chronic bronchitis," British Medical Journal, vol. 1, no. 6013, pp. 822-823, 1976.

[8] S. S. Ng, W. W. Tsang, T. H. Cheung, J. S. Chung, F. P. To, and P. C. Yu, "Walkway length, but not turning direction, determines the six-minute walk test distance in individuals with stroke," Archives of Physical Medicine and Rehabilitation, vol. 92, no. 5, pp. 806-811, 2011.

[9] R. O. Crapo, R. Casaburi, A. L. Coates et al., "ATS statement: guidelines for the six-minute walk test," The American Journal of Respiratory and Critical Care Medicine, vol. 166, no. 1, pp. 111$117,2002$.

[10] K. L. Hollands, M. A. Hollands, D. Zietz, A. M. Wing, C. Wright, and P. van Vliet, "Kinematics of turning $180^{\circ}$ during the timed up and go in stroke survivors with and without falls history," Neurorehabilitation and Neural Repair, vol. 24, no. 4, pp. 358367,2010

[11] K. L. Hollands, P. van Vliet, D. Zietz, A. Wing, C. Wright, and M. A. Hollands, "Stroke-related differences in axial body segment coordination during preplanned and reactive changes 
in walking direction," Experimental Brain Research, vol. 202, no. 3, pp. 591-604, 2010.

[12] P. S. Pohl, P. W. Duncan, S. Perera et al., "Influence of strokerelated impairments on performance in 6-minute walk test," Journal of Rehabilitation Research and Development, vol. 39, no. 4, pp. 439-444, 2002.

[13] D. F. Stroup, J. A. Berlin, S. C. Morton et al., "Meta-analysis of observational studies in epidemiology: a proposal for reporting. Meta-analysis of observational studies in epidemiology (MOOSE) group," The Journal of the American Medical Association, vol. 283, no. 15, pp. 2008-2012, 2000.

[14] World Health Organisation, WHO STEPS Stroke Manual: The WHO STEP wise Approach to Stroke Surveillance, World Health Organization, Geneva, Switzerland, 2006.

[15] L. Ada, C. M. Dean, J. M. Hall, J. Bampton, and S. Crompton, "A treadmill and overground walking program improves walking in persons residing in the community after stroke: a placebocontrolled, randomized trial," Archives of Physical Medicine and Rehabilitation, vol. 84, no. 10, pp. 1486-1491, 2003.

[16] L. Allet, B. Leemann, E. Guyen et al., "Effect of different walking aids on walking capacity of patients with poststroke hemiparesis," Archives of Physical Medicine and Rehabilitation, vol. 90, no. 8, pp. 1408-1413, 2009.

[17] C. S. Batcho, G. Stoquart, and J.-L. Thonnard, "Brisk walking can promote functional recovery in chronic stroke patients," Journal of Rehabilitation Medicine, vol. 45, no. 9, pp. 854-859, 2013.

[18] C. C. Bassile, C. Dean, B. Boden-Albala, and R. Sacco, "Obstacle training programme for individuals post stroke: feasibility study," Clinical Rehabilitation, vol. 17, no. 2, pp. 130-136, 2003.

[19] S. A. Billinger, A. E. Mattlage, A. L. Ashenden, A. A. Lentz, G. Harter, and M. A. Rippee, "Aerobic exercise in subacute stroke improves cardiovascular health and physical performance," Journal of Neurologic Physical Therapy, vol. 36, no. 4, pp. 159165, 2012.

[20] J. Blennerhassett and W. Dite, "Additional task-related practice improves mobility and upper limb function early after stroke: a randomised controlled trial," Australian Journal of Physiotherapy, vol. 50, no. 4, pp. 219-224, 2004.

[21] J. M. Blennerhassett, W. Dite, E. R. Ramage, and M. E. Richmond, "Changes in balance and walking from stroke rehabilitation to the community: a follow-up observational study," Archives of Physical Medicine and Rehabilitation, vol. 93, no. 10, pp. 1782-1787, 2012.

[22] M. G. Bowden, A. L. Behrman, R. R. Neptune, C. M. Gregory, and S. A. Kautz, "Locomotor rehabilitation of individuals with chronic stroke: difference between responders and nonresponders," Archives of Physical Medicine and Rehabilitation, vol. 94, no. 5, pp. 856-862, 2013.

[23] K. Brock, G. Haase, G. Rothacher, and S. Cotton, "Does physiotherapy based on the Bobath concept, in conjunction with a task practice, achieve greater improvement in walking ability in people with stroke compared to physiotherapy focused on structured task practice alone? A pilot randomized controlled trial," Clinical Rehabilitation, vol. 25, no. 10, pp. 903-912, 2011.

[24] C. Brogårdh, U. B. Flansbjer, and J. Lexell, "No specific effect of whole-body vibration training in chronic stroke: a double-blind randomized controlled study," Archives of Physical Medicine and Rehabilitation, vol. 93, no. 2, pp. 253-258, 2012.
[25] C. Brogårdh, U.-B. Flansbjer, and J. Lexell, "Self-reported walking ability in persons with chronic stroke and the relationship with gait performance tests," Physical Medicine and Rehabilitation, vol. 4, no. 10, pp. 734-738, 2012.

[26] S.-D. Byun, T.-D. Jung, C.-H. Kim, and Y.-S. Lee, "Effects of the sliding rehabilitation machine on balance and gait in chronic stroke patients-a controlled clinical trial," Clinical Rehabilitation, vol. 25, no. 5, pp. 408-415, 2011.

[27] S. Carda, M. Invernizzi, A. Baricich, and C. Cisari, "Casting, taping or stretching after botulinum toxin type A for spastic equinus foot: a single-blind randomized trial on adult stroke patients," Clinical Rehabilitation, vol. 25, no. 12, pp. 1119-1127, 2011.

[28] S. Carda, M. Invernizzi, G. Cognolato, E. Piccoli, A. Baricich, and C. Cisari, "Efficacy of a hip flexion assist orthosis in adults with hemiparesis after stroke," Physical Therapy, vol. 92, no. 5, pp. 734-739, 2012.

[29] S. L. Carroll, C. A. Greig, S. J. Lewis et al., "The use of pedometers in stroke survivors: are they feasible and how well do they detect steps?" Archives of Physical Medicine and Rehabilitation, vol. 93, no. 3, pp. 466-470, 2012.

[30] C. Carvalho, C. Willén, and K. S. Sunnerhagen, "Relationship between walking function and one-legged bicycling test in subjects in the later stage post-stroke," Journal of Rehabilitation Medicine, vol. 40, no. 9, pp. 721-726, 2008.

[31] C. Carvalho, K. S. Sunnerhagen, and C. Willén, "Walking performance and muscle strength in the later stage poststroke: a nonlinear relationship," Archives of Physical Medicine and Rehabilitation, vol. 94, no. 5, pp. 845-850, 2013.

[32] W. Chanruengvanich, S. Kasemkitwattana, C. Charoenyooth, S. Towanabut, and C. Pongurgsorn, "RCT: self-regulated exercise program in transient ischemic attack and minor stroke patients," Thai Journal of Nursing Research, vol.10, no. 3, pp. 165179, 2006.

[33] T. D. Da Silva, R. D. Raimundo, C. Ferreira et al., "Comparison between the six-minute walk test and the six-minute step test in post stroke patients," International Archives of Medicine, vol. 6, no. 1, article 31, 2013.

[34] J. J. Daly, K. Roenigk, J. Holcomb et al., "A randomized controlled trial of functional neuromuscular stimulation in chronic stroke subjects," Stroke, vol. 37, no. 1, pp. 172-178, 2006.

[35] J. J. Daly, J. Zimbelman, K. L. Roenigk et al., "Recovery of coordinated gait: Randomized controlled stroke trial of functional electrical stimulation (FES) versus no FES, with weightsupported treadmill and over-ground training," Neurorehabilitation and Neural Repair, vol. 25, no. 7, pp. 588-596, 2011.

[36] A. Danielsson, C. Willén, and K. S. Sunnerhagen, "Is walking endurance associated with activity and participation late after stroke?" Disability and Rehabilitation, vol. 33, no. 21-22, pp. 2053-2057, 2011.

[37] C. M. Dean, C. L. Richards, and F. Malouin, "Walking speed over 10 metres overestimates locomotor capacity after stroke," Clinical Rehabilitation, vol. 15, no. 4, pp. 415-421, 2001.

[38] K. Donovan, S. E. Lord, H. K. McNaughton, and M. Weatherall, "Mobility beyond the clinic: the effect of environment on gait and its measurement in community-ambulant stroke survivors," Clinical Rehabilitation, vol. 22, no. 6, pp. 556-563, 2008.

[39] P. Duncan, L. Richards, D. Wallace et al., "A randomized, controlled pilot study of a home-based exercise program for individuals with mild and moderate stroke," Stroke, vol. 29, no. 10, pp. 2055-2060, 1998. 
[40] P. W. Duncan, S. Studenski, L. Richards et al., "Randomized clinical trial of therapeutic exercise in subacute stroke," Stroke, vol. 34, no. 9, pp. 2173-2180, 2003.

[41] P. W. Duncan, K. J. Sullivan, A. L. Behrman et al., "Bodyweight-supported treadmill rehabilitation after stroke," The New England Journal of Medicine, vol. 364, no. 21, pp. 20262036, 2011.

[42] J. J. Eng, K. S. Chu, A. S. Dawson, C. M. Kim, and K. E. Hepburn, "Functional walk tests in individuals with stroke: relation to perceived exertion and myocardial exertion," Stroke, vol. 33, no. 3, pp. 756-761, 2002.

[43] J. J. Eng, A. S. Dawson, and K. S. Chu, "Submaximal exercise in persons with stroke: test-retest reliability and concurrent validity with maximal oxygen consumption," Archives of Physical Medicine and Rehabilitation, vol. 85, no. 1, pp. 113-118, 2004.

[44] U.-B. Flansbjer, M. Miller, D. Downham, and J. Lexell, "Progressive resistance training after stroke: effects on muscle strength, muscle tone, gait performance and perceived participation," Journal of Rehabilitation Medicine, vol. 40, no. 1, pp. 42-48, 2008.

[45] A. Forsberg and Y. Nilsagård, "Validity and reliability of the Swedish version of the activities-specific balance confidence scale in people with chronic stroke," Physiotherapy Canada, vol. 65, no. 2, pp. 141-147, 2013.

[46] S. Fritz, D. Peters, A. Merlo, and J. Donley, "Active videogaming effects on balance and mobility in individuals with chronic stroke: a randomized controlled trial," Topics in Stroke Rehabilitation, vol. 20, no. 3, pp. 218-225, 2013.

[47] G. D. Fulk, J. L. Echternach, L. Nof, and S. O'Sullivan, "Clinometric properties of the six-minute walk test in individuals undergoing rehabilitation poststroke," Physiotherapy Theory and Practice, vol. 24, no. 3, pp. 195-204, 2008.

[48] G. D. Fulk, C. Reynolds, S. Mondal, and J. E. Deutsch, "Predicting home and community walking activity in people with stroke," Archives of Physical Medicine and Rehabilitation, vol. 91, no. 10, pp. 1582-1586, 2010.

[49] C. Geroin, A. Picelli, D. Munari, A. Waldner, C. Tomelleri, and N. Smania, "Combined transcranial direct current stimulation and robot-assisted gait training in patients with chronic stroke: a preliminary comparison," Clinical Rehabilitation, vol. 25, no. 6, pp. 537-548, 2011.

[50] K. H. Gerrits, M. J. Beltman, P. A. Koppe et al., "Isometric muscle function of knee extensors and the relation with functional performance in patients with stroke," Archives of Physical Medicine and Rehabilitation, vol. 90, no. 3, pp. 480-487, 2009.

[51] T. I. Gjellesvik, B. Brurok, J. Hoff, T. Tørhaug, and J. Helgerud, "Effect of high aerobic intensity interval treadmill walking in people with chronic stroke: a pilot study with one year followup," Topics in Stroke Rehabilitation, vol. 19, no. 4, pp. 353-360, 2012.

[52] C. Globas, C. Becker, J. Cerny et al., "Chronic stroke survivors benefit from high-intensity aerobic treadmill exercise: a randomized control trial," Neurorehabilitation and Neural Repair, vol. 26, no. 1, pp. 85-95, 2012.

[53] C. D. Gordon, R. Wilks, and A. McCaw-Binns, "Effect of aerobic exercise (walking) training on functional status and healthrelated quality of life in chronic stroke survivors: a randomized controlled trial," Stroke, vol. 44, no. 4, pp. 1179-1181, 2013.

[54] J. Hidler, D. Nichols, M. Pelliccio et al., "Multicenter randomized clinical trial evaluating the effectiveness of the Lokomat in subacute stroke," Neurorehabilitation and Neural Repair, vol. 23, no. 1, pp. 5-13, 2009.
[55] H. E. Hinson, S. L. Patterson, R. F. Macko, and A. P. Goldberg, "Reduced cardiovascular fitness and ambulatory function in black and white stroke survivors," Ethnicity and Disease, vol. 17, no. 4, pp. 682-685, 2007.

[56] C. L. Hoang, J.-Y. Salle, S. Mandigout, J. Hamonet, F. MacianMontoro, and J.-C. Daviet, "Physical factors associated with fatigue after stroke: an exploratory study," Topics in Stroke Rehabilitation, vol. 19, no. 5, pp. 369-376, 2012.

[57] T. G. Hornby, D. D. Campbell, J. H. Kahn, T. Demott, J. L. Moore, and H. R. Roth, "Enhanced gait-related improvements after therapist- versus robotic-assisted locomotor training in subjects with chronic stroke: a randomized controlled study," Stroke, vol. 39, no. 6, pp. 1786-1792, 2008.

[58] S. Hwang, Y. Woo, K.-H. Kim, and K.-I. Ki, "Effects of falls experience on cognitive functions and physical activities in community-dwelling individuals with chronic stroke," International Journal of Rehabilitation Research, vol. 36, no. 2, pp. 134139, 2013.

[59] M. Iosa, G. Morone, A. Fusco et al., "Effects of walking endurance reduction on gait stability in patients with stroke," Stroke Research and Treatment, vol. 2012, Article ID 810415, 6 pages, 2012.

[60] T. W. Janssen, J. M. Beltman, P. Elich et al., "Effects of electric stimulation-assisted cycling training in people with chronic stroke," Archives of Physical Medicine and Rehabilitation, vol. 89, no. 3, pp. 463-469, 2008.

[61] H. Jin, Y. Jiang, Q. Wei, B. Wang, and G. Ma, "Intensive aerobic cycling training with lower limb weights in Chinese patients with chronic stroke: discordance between improved cardiovascular fitness and walking ability," Disability and Rehabilitation, vol. 34, no. 19, pp. 1665-1671, 2012.

[62] H.-K. Kang, Y. Kim, Y. Chung, and S. Hwang, "Effects of treadmill training with optic flow on balance and gait in individuals following stroke: randomized controlled trials," Clinical Rehabilitation, vol. 26, no. 3, pp. 246-255, 2012.

[63] C. P. Kelley, J. Childress, C. Boake, and E. A. Noser, "Overground and robotic-assisted locomotor training in adults with chronic stroke: a blinded randomized clinical trial," Disability and Rehabilitation: Assistive Technology, vol. 8, no. 2, pp. 161168, 2013.

[64] B. R. Kim, E. Y. Han, S. J. Joo, S. Y. Kim, and H. M. Yoon, "Cardiovascular fitness as a predictor of functional recovery in subacute stroke patients," Disability and Rehabilitation, vol. 36, no. 3, pp. 227-231, 2014.

[65] P. Kluding and B. Gajewski, "Lower-extremity strength differences predict activity limitations in people with chronic stroke," Physical Therapy, vol. 89, no. 1, pp. 73-81, 2009.

[66] P. M. Kluding, B. Y. Tseng, and S. A. Billinger, "Exercise and executive function in individuals with chronic stroke: a pilot study," Journal of Neurologic Physical Therapy, vol. 35, no. 1, pp. $11-17,2011$.

[67] S. S. Kuys, S. G. Brauer, and L. Ada, "Higher-intensity treadmill walking during rehabilitation after stroke in feasible and not detrimental to walking pattern or quality: a pilot randomized trial," Clinical Rehabilitation, vol. 25, no. 4, pp. 316-326, 2011.

[68] J. M. Lam, C. Globas, J. Cerny et al., "Predictors of response to treadmill exercise in stroke survivors," Neurorehabilitation and Neural Repair, vol. 24, no. 6, pp. 567-574, 2010.

[69] B. Langhammer, B. Lindmark, and J. Stanghelle, "The relation between gait velocity and static and dynamic balance in the early rehabilitation of patients with acute stroke," Advances in Physiotherapy, vol. 8, no. 2, pp. 60-65, 2006. 
[70] B. Langhammer, J. K. Stanghelle, and B. Lindmark, "Exercise and health-related quality of life during the first year following acute stroke. A randomized controlled trial," Brain Injury, vol. 22, no. 2, pp. 135-145, 2008 .

[71] B. Langhammer and J. K. Stanghelle, "Exercise on a treadmill or walking outdoors? A randomized controlled trial comparing effectiveness of two walking exercise programmes late after stroke," Clinical Rehabilitation, vol. 24, no. 1, pp. 46-54, 2010.

[72] M.-J. Lee, S. L. Kilbreath, and K. M. Refshauge, "Movement detection at the ankle following stroke is poor," The Australian Journal of Physiotherapy, vol. 51, no. 1, pp. 19-24, 2005.

[73] M. J. Lee, S. L. Kilbreath, M. F. Singh et al., "Comparison of effect of aerobic cycle training and progressive resistance training on walking ability after stroke: a randomized sham exercisecontrolled study," Journal of the American Geriatrics Society, vol. 56, no. 6, pp. 976-985, 2008.

[74] S. Lord, K. M. McPherson, H. K. McNaughton, L. Rochester, and M. Weatherall, "How feasible is the attainment of community ambulation after stroke? A pilot randomized controlled trial to evaluate community-based physiotherapy in subacute stroke," Clinical Rehabilitation, vol. 22, no. 3, pp. 215-225, 2008.

[75] M. MacKay-Lyons, A. McDonald, J. Matheson, G. Eskes, and M.-A. Klus, "Dual effects of body-weight supported treadmill training on cardiovascular fitness and walking ability early after stroke: a randomized controlled trial," Neurorehabilitation and Neural Repair, vol. 27, no. 7, pp. 644-653, 2013.

[76] R. F. Macko, F. M. Ivey, L. W. Forrester et al., “Treadmill exercise rehabilitation improves ambulatory function and cardiovascular fitness in patients with chronic stroke: a randomized, controlled trial," Stroke, vol. 36, no. 10, pp. 2206-2211, 2005.

[77] R. F. Macko, F. Benvenuti, S. Stanhope et al., "Adaptive physical activity improves mobility function and quality of life in chronic hemiparesis," Journal of Rehabilitation Research and Development, vol. 45, no. 2, pp. 323-328, 2008.

[78] N. Maeda, J. Kato, Y. Azuma et al., "Energy expenditure and walking ability in stroke patients: their improvement by anklefoot orthoses," Isokinetics and Exercise Science, vol. 17, no. 2, pp. 57-62, 2009.

[79] D. Marsden, R. Quinn, N. Pond et al., "A multidisciplinary group programme in rural settings for community-dwelling chronic stroke survivors and their carers: a pilot randomized controlled trial," Clinical Rehabilitation, vol. 24, no. 4, pp. 328$341,2010$.

[80] N. E. Mayo, M. J. Mackay-Lyons, S. C. Scott, C. Moriello, and J. Brophy, "A randomized trial of two home-based exercise programmes to improve functional walking post-stroke," Clinical Rehabilitation, vol. 27, no. 7, pp. 659-671, 2013.

[81] J. Mehrholz, K. Rutte, and M. Pohl, "Jump training is feasible for nearly ambulatory patients after stroke," Clinical Rehabilitation, vol. 20, no. 5, pp. 406-412, 2006.

[82] J. Mehrholz, K. Wagner, K. Rutte, D. Meißner, and M. Pohl, "Predictive validity and responsiveness of the functional ambulation category in hemiparetic patients after stroke," Archives of Physical Medicine and Rehabilitation, vol. 88, no. 10, pp. 13141319, 2007.

[83] K. Michael, A. P. Goldberg, M. S. Treuth, J. Beans, P. Normandt, and R. F. MacKo, "Progressive adaptive physical activity in stroke improves balance, gait, and fitness: preliminary results," Topics in Stroke Rehabilitation, vol. 16, no. 2, pp. 133-139, 2009.

[84] C. Miklitsch, C. Krewer, S. Freivogel, and D. Steube, "Effects of a predefined mini-trampoline training programme on balance, mobility and activities of daily living after stroke: a randomized controlled pilot study," Clinical Rehabilitation, vol. 27, no. 10, pp. 939-947, 2013.

[85] C. Moriello, L. Finch, and N. E. Mayo, "Relationship between muscle strength and functional walking capacity among people with stroke," Journal of Rehabilitation Research and Development, vol. 48, no. 3, pp. 267-275, 2011.

[86] S. Mudge, P. A. Barber, and N. S. Stott, "Circuit-based rehabilitation improves gait endurance but not usual walking activity in chronic stroke: a randomized controlled trial," Archives of Physical Medicine and Rehabilitation, vol. 90, no. 12, pp. 19891996, 2009.

[87] S. Mudge and N. S. Stott, "Timed walking tests correlate with daily step activity in persons with stroke," Archives of Physical Medicine and Rehabilitation, vol. 90, no. 2, pp. 296-301, 2009.

[88] M. A. Muren, M. Hütler, and J. Hooper, "Functional capacity and health-related quality of life in individuals post stroke," Topics in Stroke Rehabilitation, vol. 15, no. 1, pp. 51-58, 2008.

[89] A. Murtezani, H. Hundozi, S. Gashi, T. Osmani, V. Krasniqi, and B. Rama, "Factors associated with reintegration to normal living after stroke," Medicinski Arhiv, vol. 63, no. 4, pp. 216-219, 2009.

[90] S. S. Ng and C. W. Hui-Chan, "The timed up \& go test: its reliability and association with lower-limb impairments and locomotor capacities in people with chronic stroke," Archives of Physical Medicine and Rehabilitation, vol. 86, no. 8, pp. 16411647, 2005.

[91] S. S. M. Ng and C. W. Y. Hui-Chan, "Does the use of TENS increase the effectiveness of exercise for improving walking after stroke? A randomized controlled clinical trial," Clinical Rehabilitation, vol. 23, no. 12, pp. 1093-1103, 2009.

[92] S. S. Ng and C. W. Hui-Chan, "Contribution of ankle dorsiflexor strength to walking endurance in people with spastic hemiplegia after stroke," Archives of Physical Medicine and Rehabilitation, vol. 93, no. 6, pp. 1046-1051, 2012.

[93] K. J. Nolan, K. K. Savalia, A. H. Lequerica, and E. P. Elovic, "Objective assessment of functional ambulation in adults with hemiplegia using ankle foot orthotics after stroke," $P M$ and $R$, vol. 1, no. 6, pp. 524-529, 2009.

[94] O. A. Olawale, S. I. Jaja, C. N. Anigbogu, K. O. Appiah-Kubi, and D. Jones-Okai, "Exercise training improves walking function in an African group of stroke survivors: a randomized controlled trial," Clinical Rehabilitation, vol. 25, no. 5, pp. 442-450, 2011.

[95] M. M. Ouellette, N. K. LeBrasseur, J. F. Bean et al., "Highintensity resistance training improves muscle strength, selfreported function, and disability in long-term stroke survivors," Stroke, vol. 35, no. 6, pp. 1404-1409, 2004.

[96] J. C. Outermans, R. P. Van Peppen, H. Wittink, T. Takken, and G. Kwakkel, "Effects of a high-intensity task-oriented training on gait performance early after stroke: a pilot study," Clinical Rehabilitation, vol. 24, no. 11, pp. 979-987, 2010.

[97] A. C. Ovando, S. M. Michaelsen, T. de Carvalho, and V. Herber, "Evaluation of cardiopulmonary fitness in individuals with hemiparesis after cerebrovascular accident," Arquivos Brasileiros de Cardiologia, vol. 96, no. 2, pp. 140-146, 2011.

[98] M. Y. C. Pang, J. J. Eng, A. S. Dawson, H. A. McKay, and J. E. Harris, "A community-based fitness and mobility exercise program for older adults with chronic stroke: a randomized, controlled trial," Journal of the American Geriatrics Society, vol. 53, no. 10, pp. 1667-1674, 2005.

[99] H.-J. Park, D.-W. Oh, S.-Y. Kim, and J.-D. Choi, "Effectiveness of community-based ambulation training for walking function 
of post-stroke hemiparesis: a randomized controlled pilot trial," Clinical Rehabilitation, vol. 25, no. 5, pp. 451-459, 2011.

[100] S. L. Patterson, L. W. Forrester, M. M. Rodgers et al., "Determinants of walking function after stroke: differences by deficit severity," Archives of Physical Medicine and Rehabilitation, vol. 88, no. 1, pp. 115-119, 2007.

[101] S. L. Patterson, M. M. Rodgers, R. F. Macko, and L. W. Forrester, "Effect of treadmill exercise training on spatial and temporal gait parameters in subjects with chronic stroke: a preliminary report," Journal of Rehabilitation Research and Development, vol. 45, no. 2, pp. 221-228, 2008.

[102] S. H. Peurala, I. M. Tarkka, K. Pitkänen, and J. Sivenius, “The effectiveness of body weight-supported gait training and floor walking in patients with chronic stroke," Archives of Physical Medicine and Rehabilitation, vol. 86, no. 8, pp. 1557-1564, 2005.

[103] J. C. Polese, M. B. Pinheiro, C. D. C. M. Faria, R. R. Britto, V. F. Parreira, and L. F. Teixeira-Salmela, "Strength of the respiratory and lower limb muscles and functional capacity in chronic stroke survivors with different physical activity levels," Brazilian Journal of Physical Therapy, vol. 17, no. 5, pp. 487-493, 2013.

[104] D. Pradon, N. Roche, L. Enette, and R. Zory, "Relationship between lower limb muscle strength and 6-minute walk test performance in stroke patients," Journal of Rehabilitation Medicine, vol. 45, no. 1, pp. 105-108, 2013.

[105] M. H. Rabadi, P. L. Coar, M. Lukin, M. Lesser, and J. P. Blass, "Intensive nutritional supplements can improve outcomes in stroke rehabilitation," Neurology, vol. 71, no. 23, pp. 1856-1861, 2008.

[106] D. Rand, J. J. Eng, P.-F. Tang, J.-S. Jeng, and C. Hung, "How active are people with stroke? Use of accelerometers to assess physical activity," Stroke, vol. 40, no. 1, pp. 163-168, 2009.

[107] A. S. Ryan, A. Buscemi, L. Forrester, C. E. Hafer-Macko, and F. M. Ivey, "Atrophy and intramuscular fat in specific muscles of the thigh: Associated weakness and hyperinsulinemia in stroke survivors," Neurorehabilitation and Neural Repair, vol. 25, no. 9, pp. 865-872, 2011.

[108] C. M. Ryan, M. Bayley, R. Green, B. J. Murray, and T. D. Bradley, "Influence of continuous positive airway pressure on outcomes of rehabilitation in stroke patients with obstructive sleep apnea," Stroke, vol. 42, no. 4, pp. 1062-1067, 2011.

[109] N. M. Salbach, N. E. Mayo, S. Wood-Dauphinee, J. A. Hanley, C. L. Richards, and R. Côté, "A task-orientated intervention enhances walking distance and speed in the first year post stroke: a randomized controlled trial," Clinical Rehabilitation, vol. 18, no. 5, pp. 509-519, 2004.

[110] N. M. Salbach, D. Brooks, J. Romano, L. Woon, and T. E. Dolmage, "Cardiorespiratory responses during the 6-minute walk and ramp cycle ergometer tests and their relationship to physical activity in stroke," Neurorehabilitation and Neural Repair, vol. 28, no. 2, pp. 111-119, 2014.

[111] A. A. Schmid, M. Van Puymbroeck, P. A. Altenburger et al., "Balance and balance self-efficacy are associated with activity and participation after stroke: a cross-sectional study in people with chronic stroke," Archives of Physical Medicine and Rehabilitation, vol. 93, no. 6, pp. 1101-1107, 2012.

[112] K. Severinsen, J. K. Jakobsen, K. Overgaard, and H. Andersen, "Normalized muscle strength, aerobic capacity, and walking performance in chronic stroke: a population-based study on the potential for endurance and resistance training," Archives of Physical Medicine and Rehabilitation, vol. 92, no. 10, pp. 16631668, 2011.
[113] K. M. Sibley, A. Tang, D. Brooks, and W. E. McIlroy, "Effects of extended effortful activity on spatio-temporal parameters of gait in individuals with stroke," Gait and Posture, vol. 27, no. 3, pp. 387-392, 2008.

[114] L. A. Simpson, W. C. Miller, and J. J. Eng, "Effect of stroke on fall rate, location and predictors: a prospective comparison of older adults with and without stroke," PLoS ONE, vol. 6, no. 4, Article ID e19431, 2011.

[115] D. K. A. Singh, N. A. Mohd Nordin, N. A. A. Aziz, B. K. Lim, and L. C. Soh, "Effects of substituting a portion of standard physiotherapy time with virtual reality games among community-dwelling stroke survivors," BMC Neurology, vol. 13, article 199, 2013.

[116] A. D. Stookey, M. G. McCusker, J. D. Sorkin et al., "Test-retest reliability of portable metabolic monitoring after disabling stroke," Neurorehabilitation and Neural Repair, vol. 27, no. 9, pp. 872-877, 2013.

[117] A. D. Stookey, L. I. Katzel, G. Steinbrenner, M. Shaughnessy, and F. M. Ivey, "The short physical performance battery as a predictor of functional capacity after stroke," Journal of Stroke and Cerebrovascular Diseases, vol. 23, no. 1, pp. 130-135, 2014.

[118] M. Stuart, F. Benvenuti, R. MacKo et al., "Community-based adaptive physical activity program for chronic stroke: feasibility, safety, and efficacy of the empoli model," Neurorehabilitation and Neural Repair, vol. 23, no. 7, pp. 726-734, 2009.

[119] K. J. Sullivan, D. A. Brown, T. Klassen et al., "Effects of taskspecific locomotor and strength training in adults who were ambulatory after stroke: results of the STEPS randomized clinical trial," Physical Therapy, vol. 87, no. 12, pp. 1580-1602, 2007.

[120] A. Tang, K. M. Sibley, M. T. Bayley, W. E. McIlroy, and D. Brooks, "Do functional walk tests reflect cardiorespiratory fitness in sub-acute stroke?" Journal of NeuroEngineering and Rehabilitation, vol. 3, article 23, 2006.

[121] A. Tang, K. M. Sibley, S. G. Thomas et al., "Effects of an aerobic exercise program on aerobic capacity, spatiotemporal gait parameters, and functional capacity in subacute stroke," Neurorehabilitation and Neural Repair, vol. 23, no. 4, pp. 398-406, 2009.

[122] D. Tanne, R. Tsabari, O. Chechk et al., "Improved exercise capacity in patients after minor ischemic stroke undergoing a supervised exercise training program," Israel Medical Association Journal, vol. 10, no. 2, pp. 113-116, 2008.

[123] A. Toledano-Zarhi, D. Tanne, E. Carmeli, and M. Katz-Leurer, "Feasibility, safety and efficacy of an early aerobic rehabilitation program for patients after minor ischemic stroke: a pilot randomized controlled trial," NeuroRehabilitation, vol. 28, no. 2, pp. 85-90, 2011.

[124] B. Y. Tseng and P. Kluding, "The relationship between fatigue, aerobic fitness, and motor control in people with chronic stroke: a pilot study," Journal of Geriatric Physical Therapy, vol. 32, no. 3, pp. 97-102, 2009.

[125] M. van Bloemendaal, A. M. Kokkeler, and I. G. van de Port, "The shuttle walk test: a new approach to functional walking capacity measurements for patients after stroke?" Archives of Physical Medicine and Rehabilitation, vol. 93, no. 1, pp. 163-166, 2012.

[126] I. G. L. van de Port, L. E. G. Wevers, E. Lindeman, and G. Kwakkel, "Effects of circuit training as alternative to usual physiotherapy after stroke: randomised controlled trial," British Medical Journal, vol. 344, no. 7859, Article ID e2672, 2012. 
[127] J. L. Verheijde, F. White, J. Tompkins et al., "Reliability, validity, and sensitivity to change of the lower extremity functional scale in individuals affected by stroke," Physical Medicine and Rehabilitation, vol. 5, no. 12, pp. 1019-1025, 2013.

[128] R. Verma, K. Arya, R. K. Garg, and T. Singh, “Task-oriented circuit class training program with motor imagery for gait rehabilitation in poststroke patients: a randomized controlled trial," Topics in Stroke Rehabilitation, vol. 18, supplement 1, pp. 620-632, 2011.

[129] K. P. Westlake and C. Patten, "Pilot study of Lokomat versus manual-assisted treadmill training for locomotor recovery post-stroke," Journal of NeuroEngineering and Rehabilitation, vol. 6, no. 1, article 18, 2009.

[130] L. E. G. Wevers, G. Kwakkel, and I. G. L. van de Port, "Is outdoor use of the six-minute walk test with a global positioning system in stroke patients' own neighbourhoods reproducible and valid?" Journal of Rehabilitation Medicine, vol. 43, no. 11, pp. 1027-1031, 2011.

[131] J. H. White, B. L. Bynon, J. Marquez, A. Sweetapple, and M. Pollack, "Masterstroke: a pilot group stroke prevention program for community dwelling stroke survivors," Disability and Rehabilitation, vol. 35, no. 11, pp. 931-938, 2013.

[132] K. Wing, J. V. Lynskey, and P. R. Bosch, "Whole-body intensive rehabilitation is feasible and effective in chronic stroke survivors: a retrospective data analysis," Topics in Stroke Rehabilitation, vol. 15, no. 3, pp. 247-255, 2008.

[133] Y.-R. Yang, R.-Y. Wang, K.-H. Lin, M.-Y. Chu, and R.-C. Chan, "Task-oriented progressive resistance strength training improves muscle strength and functional performance in individuals with stroke," Clinical Rehabilitation, vol. 20, no. 10, pp. 860-870, 2006.

[134] H.-C. Yang, C.-L. Lee, R. Lin et al., "Effect of biofeedback cycling training on functional recovery and walking ability of lower extremity in patients with stroke," The Kaohsiung Journal of Medical Sciences, vol. 30, no. 1, pp. 35-42, 2014.

[135] J. Yiu, W. C. Miller, J. J. Eng, and Y. Liu, "Longitudinal analysis of balance confidence in individuals with stroke using a multilevel model for change," Neurorehabilitation and Neural Repair, vol. 26, no. 8, pp. 999-1006, 2012.

[136] K. R. Zalewski and L. Dvorak, "Barriers to physical activity between adults with stroke and their care partners," Topics in Stroke Rehabilitation, vol. 18, no. 1, pp. 666-675, 2011.

[137] A. M. E. E. Zedlitz, T. C. M. Rietveld, A. C. Geurts, and L. Fasotti, "Cognitive and graded activity training can alleviate persistent fatigue after stroke: a randomized, controlled trial," Stroke, vol. 43, no. 4, pp. 1046-1051, 2012.

[138] C. Carvalho, K. S. Sunnerhagen, and C. Willén, "Walking speed and distance in different environments of subjects in the later stage post-stroke," Physiotherapy Theory and Practice, vol. 26, no. 8, pp. 519-527, 2010

[139] K. Holland, P. van Vliet, and T. Pelton, "Interventions for improving coordination of axial segments and lower limbs during walking following stroke," Gait \& Posture, vol. 35, no. 3, pp. 349-359, 2012.

[140] T. Lam and K. Luttmann, "Turning capacity in ambulatory individuals poststroke," American Journal of Physical Medicine \& Rehabilitation, vol. 88, no. 11, pp. 873-883, 2009.

[141] C. D. Faria, L. F. Teixeira-Salmela, and S. Nadeau, "Effects of the direction of turning on the Timed Up \& Go test with stroke subjects," Topics in Stroke Rehabilitation, vol. 16, no. 3, pp. 196206, 2009.
[142] T. H. M. Heung and S. S. M. Ng, "Effect of seat height and turning direction on the timed up and go test scores of people after stroke," Journal of Rehabilitation Medicine, vol. 41, no. 9, pp. 719-722, 2009.

[143] R. W. Bohannon, "Six-minute walk test: a meta-analysis of data from apparently healthy elders," Topics in Geriatric Rehabilitation, vol. 23, no. 2, pp. 155-160, 2007. 


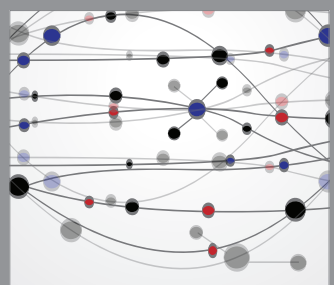

The Scientific World Journal
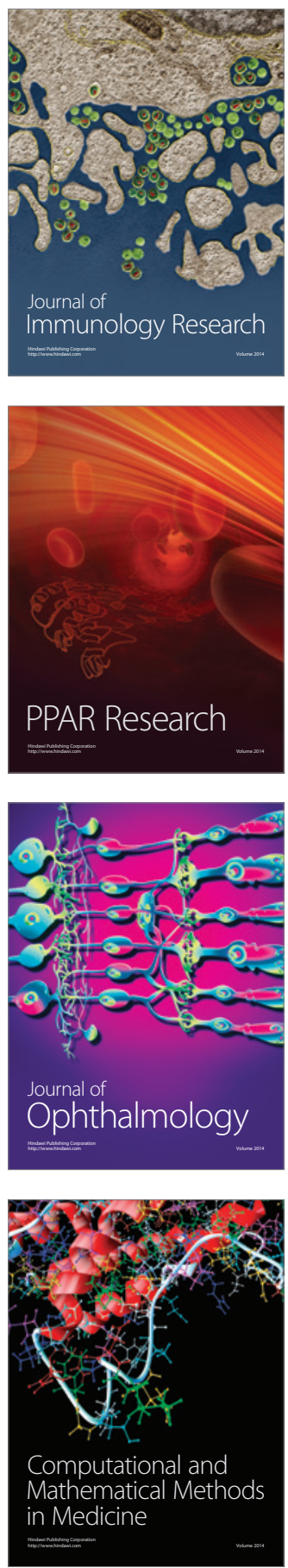

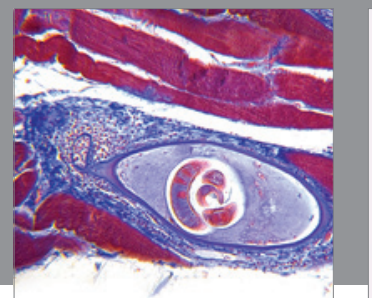

Gastroenterology

Research and Practice
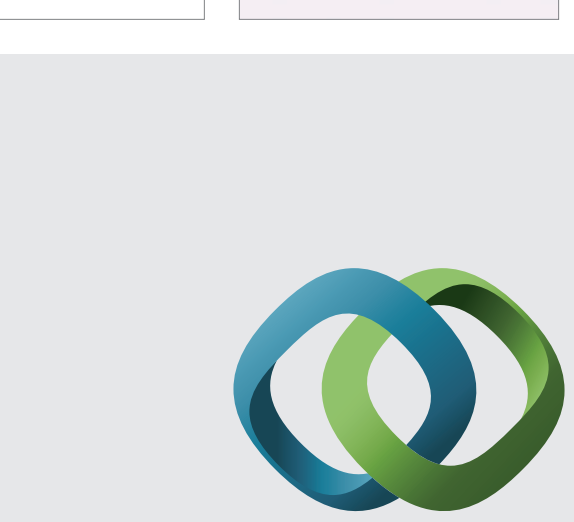

\section{Hindawi}

Submit your manuscripts at

http://www.hindawi.com
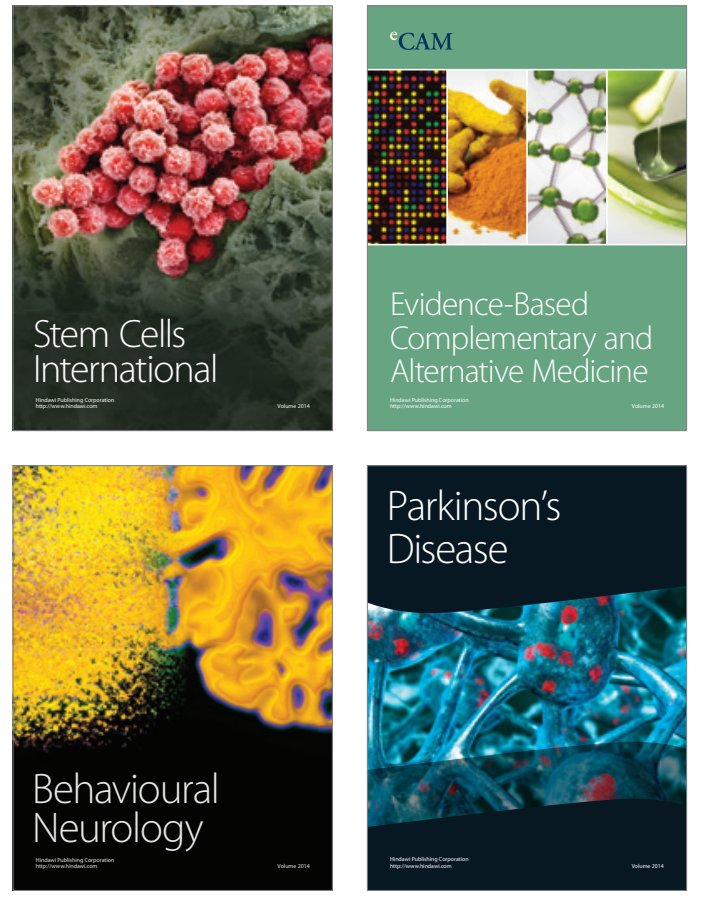
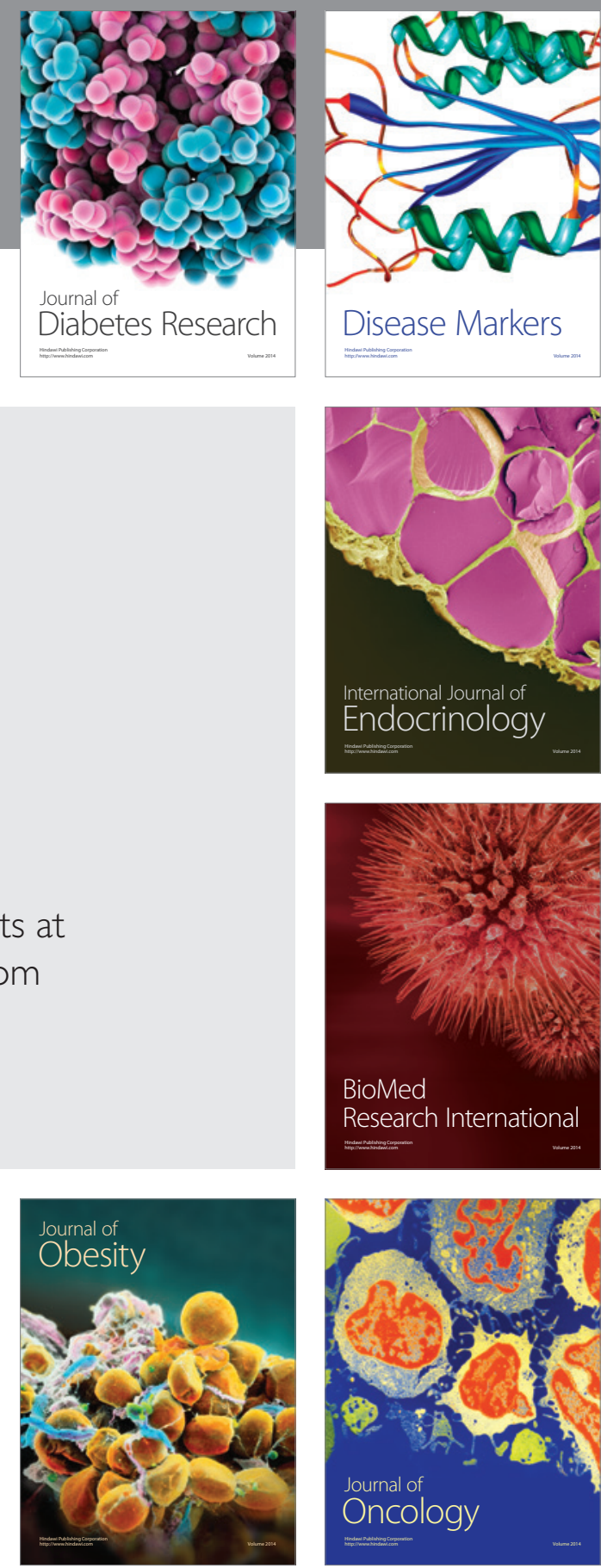

Disease Markers
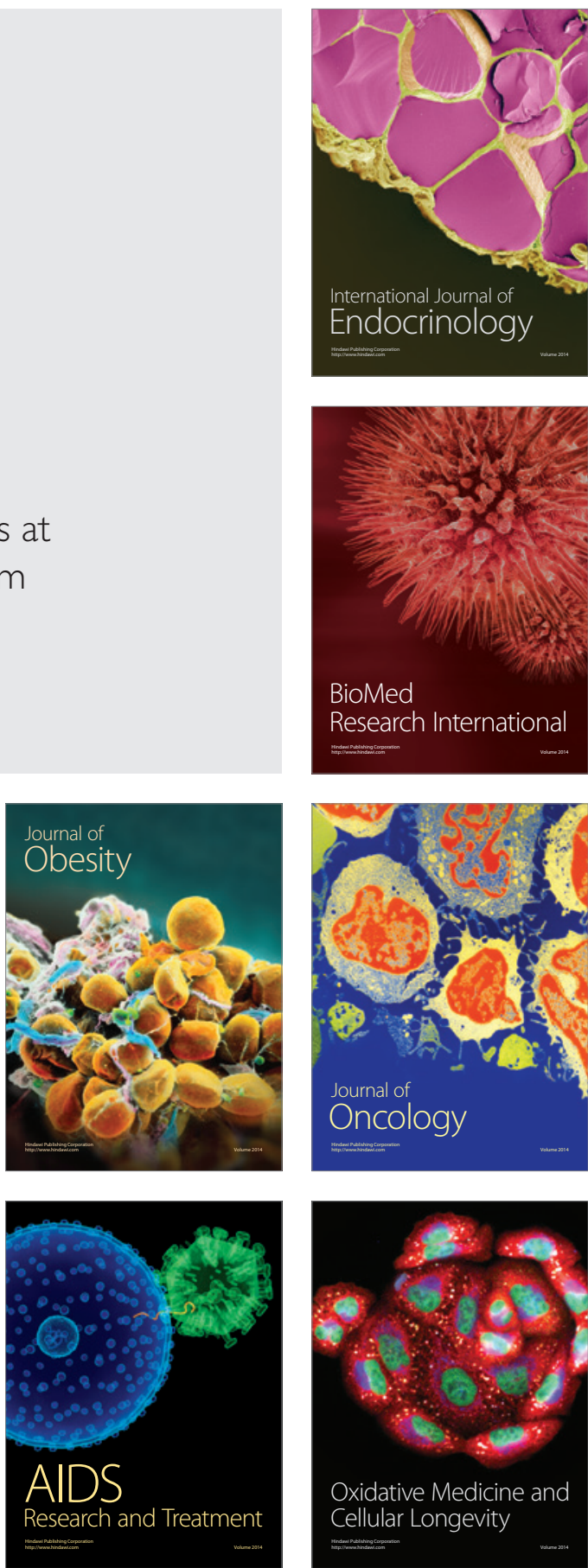